\title{
A VON NEUMANN ALGEBRA CHARACTERIZATION OF PROPERTY (T) FOR GROUPOIDS
}

\author{
MARTINO LUPINI
}

\begin{abstract}
For an arbitrary discrete probability-measure-preserving groupoid $G$, we provide a characterization of property (T) for $G$ in terms of the groupoid von Neumann algebra $L(G)$. More generally, we obtain a characterization of relative property (T) for a subgroupoid $H \subset G$ in terms of the inclusion $L(H) \subset L(G)$.
\end{abstract}

\section{INTRODUCTION}

Property $(\mathrm{T})$ for countable probability-measure-preserving (pmp) equivalence relations has been introduced by Zimmer in [22]. The natural generalization to discrete pmp groupoids has been studied by Anantharaman-Delaroche in [2]. In view of the importance of property $(\mathrm{T})$ in the setting of operator algebras, and the key role it plays in Popa's deformation/rigidity theory, it is valuable to have a characterization of property (T) for a discrete pmp groupoid $G$ solely in terms of the inclusion $L^{\infty}\left(G^{0}\right) \subset L(G)$, where $L(G)$ is the groupoid von Neumann algebra of $G$ and $G^{0}$ is the unit space of $G$. Such a characterization has been established by Connes and Jones in the case when $G$ is a countable discrete group with infinite conjugacy classes, in which case $L(G)$ is a $\mathrm{II}_{1}$ factor. They showed in [5] that, under those assumptions, $G$ has property (T) if and only if $L(G)$ has property (T) in the sense defined therein. In the case when $G$ is an ergodic $\mathrm{II}_{1}$ equivalence relation, in which case $L(G)$ is a $\mathrm{II}_{1}$ factor and $L^{\infty}\left(G^{0}\right)$ is a Cartan subalgebra of $L(G)$, such a characterization has been established by Popa in [16]. It is shown there that, under those assumptions, $G$ has property (T) as defined by Zimmer if and only if the inclusion $L\left(G^{0}\right) \subset L(G)$ is co-rigid in the sense of [17, Remark 2.6.1]. The purpose of this paper is to provide a common generalization of such characterizations, applicable to an arbitrary discrete pmp groupoid $G$.

More generally, given a subgroupoid $H$ of $G$, we consider the natural notion of relative property (T) of $H$ in $G$, generalizing the usual notion for groups. We then obtain a characterization of relative property (T) of $H$ in $G$ in terms of the inclusion $L^{\infty}(X) \subset L(H) \subset L(G)$, where $X$ is the common unit space of $G$ and $H$. Again, such a characterization is applicable to an arbitrary pair of discrete pmp groupoids. In the case of groups, relative property $(\mathrm{T})$ has been characterized in terms of group von Neumann algebra by Popa in [17, Proposition 5.1]. It is shown there that the pair $H \leq G$ has property (T) if and only if the inclusion $L(H) \subset L(G)$ is rigid in the sense defined therein; see [17, Definition 4.2].

More generally, we consider a notion of property (T) for a triple $K \leq H \leq G$ consisting of a discrete pmp groupoid $G$ together with nested subgroupoid $K, H$. Such a notion has been considered in the setting of groups in [7, Definition 2.3]. It subsumes property (T) for a pair $H \leq G$ in the case when $K$ is the trivial subgroupoid of $G$, i.e. the unit space. Again we obtain, for an arbitrary such triple, a characterization of property (T) in terms of the inclusions $L^{\infty}(X) \subset L(K) \subset L(H) \subset L(G)$. We also provide a cohomological characterization of such a notion, and in particular of the notion of relative property $(\mathrm{T})$. In the case of property $(\mathrm{T})$ for a single groupoid, such a characterization has been obtained by Anantharaman-Delaroche in [2].

Date: August 8, 2017.

2000 Mathematics Subject Classification. Primary 20L05, 46L10; Secondary 37A15.

Key words and phrases. Groupoid, von Neumann algebra, property (T), Kazhdan groupoid, bimodule, cohomology, representation.

The author was partially supported by the NSF Grant DMS-1600186. 
The rest of this paper is divided into two sections, apart from this introduction. In Section 2 we recall the fundamental notions and definitions concerning groupoids to be used in the rest of the paper, introduce the notion of property $(\mathrm{T})$ for triples of groupoids, and obtain the cohomological characterization mentioned above. In Section 3 we obtain a von Neumann algebra characterization of property ( $\mathrm{T}$ ) for triples of groupoids, in terms of the groupoid von Neumann algebras.

Throughout the paper, we follow the convention that scalar products in Hilbert spaces are linear in the second variable and conjugate-linear in the first variable.

\section{Property (T) FOR GROUPOIDS}

2.1. Groupoids. A groupoid is, briefly, a small category $G$ where every morphism is invertible. In this case, the objects of $G$ are also called units, and the set of units is denoted by $G^{0}$. The morphisms in $G$ are also called arrows. As it is customary, we canonically identify every object with the corresponding identity arrow. This allows one to regard $G^{0}$ as a subset of $G$. There are canonical source and range maps $s, r: G \rightarrow G^{0}$ that map each arrow $\gamma$ in $G$ to the objects $s(\gamma), r(\gamma) \in G^{0}$ such that $\gamma$ is an arrow from $s(\gamma)$ to $r(\gamma)$. A pair of arrows $(\gamma, \rho)$ is composable if $s(\gamma)=r(\rho)$. The set of pairs of composable arrows of $G$ is denoted by $G^{2}$. One can then regard composition of arrows as a function $G^{2} \rightarrow G$, $(\gamma, \rho) \mapsto \gamma \rho$. Since by assumption every arrow of $G$ is invertible, one can also consider the function $G \rightarrow G, \gamma \mapsto \gamma^{-1}$ that maps each arrow to its inverse. In the following, given subsets $A, B$ of $G$, we let $A B$ be the set of arrows $\gamma \rho$ for $(\gamma, \rho) \in G^{2} \cap(A \times B)$. If $\gamma \in G$, we also write $A \gamma$ and $\gamma A$ for $A\{\gamma\}$ and $\{\gamma\} A$, respectively. Consistently, if $x$ is a unit of $G$, then $x A$ is the set of arrows in $A$ with range $x$, and $A x$ is the set of arrows in $A$ with source $x$.

A Borel groupoid is a groupoid $G$ endowed with a standard Borel structure such that the set of objects is Borel, and composition and inversion of arrows are Borel maps. A countable Borel groupoid is a standard Borel groupoid such that $x G$ and $G x$ are countable sets for every $x \in G^{0}$ or, equivalently, source and range maps are countable-to-one. In the following we will tacitly use classical Borel selection theorem for countable-to-one Borel maps as can be found in [13, Section 18.C]. A discrete probabilitymeasure-preserving (pmp) groupoid is a pair $(G, \mu)$ where $G$ is a countable Borel groupoid and $\mu$ is a Borel probability measure on $G^{0}$ satisfying

$$
\int_{x \in G^{0}}|x A| d \mu(x)=\int_{x \in G^{0}}|A x| d \mu(x)
$$

for every Borel subset $A$ of $G^{0}$. In such a case this expression defines an extension of $\mu$ to a $\sigma$-finite Borel measure defined on the whole of $G$. In the following we regard a discrete pmp groupoid $G$ as a measure space endowed with such a measure. One can also define a canonical measure on $G^{2}$ given by

$$
\mu_{G^{2}}(A)=\int_{x \in G^{0}}(A \cap(G x \times x G)) d \mu(x) .
$$

Given a non-null Borel subset $A$ of $G^{0}$ one can define the reduction $\left.G\right|_{A}$ to be the groupoid $A G A$ with set of objects $A$ endowed with the measure $\mu_{A}:=\frac{1}{\mu(A)} \mu$. Such a reduction is called inessential if $A$ is conull. In the following, we identify two discrete pmp groupoids whenever they have isomorphic inessential reductions. A Borel subset $A$ of $G$ is invariant if $r(G A)=A$. The groupoid $G$ is ergodic if every invariant set $A \subset G^{0}$ is either null or conull.

Suppose that $G$ is a discrete pmp groupoid, and $H$ is a standard Borel groupoid. A homomorphism from $G$ to $H$ is a Borel map $f: G \rightarrow H$ satisfying $s(f(\gamma))=f(s(\gamma))$ and $r(f(\gamma))=f(r(\gamma))$ for a.e. $\gamma \in G$, and $f(\gamma \rho)=f(\gamma) f(\rho)$ for a.e. $(\gamma, \rho) \in G^{2}$. This is equivalent to the assertion that there exists a conull Borel subset $A$ of $G^{0}$ such that $s(f(\gamma))=f(s(\gamma)), r(f(\gamma))=f(r(\gamma))$, and $f(\gamma \rho)=f(\gamma) f(\rho)$ for ever $\gamma, \rho \in A G A$ [18, Lemma 5.2]. A subgroupoid of $G$ is a Borel subset $H$ of $G$ which is also a groupoid, such that $G$ and $H$ have the same unit space $(X, \mu)$, and the inclusion map $H \subset G$ is a homomorphism from $H$ to $G$.

A (Borel) bisection of a discrete pmp groupoid $G$ is a Borel subset $t$ of $G$ such that $x t$ and $t x$ have size at most 1 for every $x \in G^{0}$. Borel bisections naturally form an inverse semigroup with respect to 
the operation

$$
\left(t_{0}, t_{1}\right) \mapsto t_{0} t_{1}=\left\{\gamma \rho:(\gamma, \rho) \in\left(t_{0} \times t_{1}\right) \cap G^{2}\right\} .
$$

The full pseudogroup $[[G]]$ of $G$ is the inverse semigroup consisting of Borel bisections modulo the relation of being equal almost everywhere. In the following we will always identify Borel bisections when they agree almost everywhere. The full group $[G]$ is the subset of $[[G]]$ consisting of the Borel bisections $t$ such that $t t^{-1}=t^{-1} t=G^{0}$. This is a Polish group with respect to the topology induced by the metric $d\left(t_{0}, t_{1}\right)=\mu\left(t_{0} \triangle t_{1}\right)$. A countable subgroup $\Gamma$ of $[G]$ covers $G$ if $G=\bigcup \Gamma$. If $G$ is ergodic and $A, B$ are Borel subsets of $G^{0}$, then $\mu(A)=\mu(B)$ if and only if there exists $t \in[G]$ such that $B=r(t A)$.

Clearly, any countable discrete group is, in particular, a discrete pmp groupoids. Indeed, these are precisely the discrete pmp groupoids whose unit space contains a single element. At the opposite end of the spectrum, every countable pmp equivalence relation is a discrete pmp groupoid. Indeed, these are precisely the discrete pmp groupoids $G$ which are principal, in the sense that the function $G \rightarrow G^{0} \times G^{0}, \gamma \mapsto(r(\gamma), s(\gamma))$ is one-to-one. Thus, the class of discrete pmp groupoids subsumes both countable discrete groups and countable pmp equivalence relations.

2.2. Representations of groupoids. Suppose that $X$ is a standard probability space. A standard Borel space fibered over $X$ is a standard Borel space $Z$ endowed with a distinguished Borel map $p: Z \rightarrow X$. In this case we let, for $x \in X, Z_{x}:=p^{-1}\{x\}$ be the corresponding fiber over $x$. We denote the space $Z$ also by $\bigsqcup_{x \in X} Z_{x}$. Given two standard Borel spaces $Z, Z^{\prime}$ fibered over $X$ one can define the fibered product

$$
Z * Z^{\prime}=\left\{\left(z, z^{\prime}\right) \in Z_{x} \times Z_{x}^{\prime}: x \in X\right\} \subset Z \times Z^{\prime},
$$

which is still a standard Borel space fibered over $X$. A fibered map $f$ from $Z$ to $Z$ ' is a Borel function that maps $Z_{x}$ to $Z_{x}^{\prime}$ for $x \in X$. If $Y$ is a standard Borel space, when we regard $Y \times X$ as a standard Borel space fibered over $X$ with respect to the product Borel structure and the projection to the second factor. In particular, we regard $X$ as a standard Borel space fibered over itself via the identity map. A section $\sigma$ for a standard Borel space $Z$ fibered over $X$ is a fibered map from $X$ to $Z$. In this case, we let $\sigma_{x}$ be the value of $\sigma$ at $x \in X$.

A (Borel, complex) Hilbert bundle over $X$ is a standard Borel space $\mathcal{H}$ fibered over $X$ endowed fibered functions $0: X \rightarrow \mathcal{H}$ (zero section), $+: \mathcal{H} * \mathcal{H} \rightarrow \mathcal{H}$ (sum), and $\mathbb{C} \times \mathcal{H} \rightarrow \mathcal{H}$ (scalar multiplication) that define on each fiber $\mathcal{H}_{x}$ for $x \in X$ a (complex) vector space structure, and such that there exists a sequence of sections $\left(\sigma_{n}\right)_{n \in \mathbb{N}}$ of $\mathcal{H}$ such that $\left\{\sigma_{n, x}: n \in \mathbb{N}\right\}$ has dense linear span in $\mathcal{H}_{x}$. The Gram-Schmidt orthonormalization process shows that one can furthermore assume that $\left\{\sigma_{n, x}: n \in \mathbb{N}\right\}$ is an orthonormal basis of $\mathcal{H}_{x}$ for $x \in X$. In this case, we call $\left(\sigma_{n}\right)_{n \in \mathbb{N}}$ an orthonormal basic sequence for $\mathcal{H}$. The unitary groupoid $U(\mathcal{H})$ is the groupoid consisting of the unitary operators $U: \mathcal{H}_{x} \rightarrow \mathcal{H}_{y}$ for $x, y \in X$. This is a standard Borel groupoid when endowed with the standard Borel structure generated by the source and range maps together with the functions $\left(U: \mathcal{H}_{x} \rightarrow \mathcal{H}_{y}\right) \mapsto\left\langle\sigma_{n, y}, U \sigma_{m, x}\right\rangle$ for $n, m \in \mathbb{N}$. The unit space of $U(\mathcal{H})$ can be identified with $X$. One can also consider the space $L^{2}(X, \mathcal{H})$ of sections for $\mathcal{H}$ satisfying

$$
\int\left\|\xi_{x}\right\|^{2} d \mu(x)<+\infty
$$

identified when they agree almost everywhere. This is a Hilbert space with respect to the inner product

$$
\langle\xi, \eta\rangle=\int\left\langle\xi_{x}, \eta_{x}\right\rangle d \mu(x)
$$

for $\xi, \eta \in L^{2}(X, \mathcal{H})$.

Suppose that $G$ is a discrete pmp groupoid, and $\mathcal{H}$ is a (complex) Hilbert bundle over $G^{0}$. A (unitary) representation of $\pi$ on $\mathcal{H}$ is a homomorphism from $G$ to $U(\mathcal{H})$ that is the identity on the unit space. An invariant sub-bundle of $\mathcal{H}$ is a Borel subset $\mathcal{K}$ of $\mathcal{H}$ such that $\mathcal{K}_{x}$ is a subspace of $\mathcal{H}$ for a.e. $x \in G^{0}$, and $\pi_{\gamma}$ maps $\mathcal{K}_{s(\gamma)}$ onto $\mathcal{K}_{r(\gamma)}$ for a.e. $\gamma \in G$. When $G$ is ergodic, the Borel function $x \mapsto \operatorname{dim} \mathcal{K}_{x}$ is constant almost everywhere, one can define its constant value to be the dimension of 
$\mathcal{K}$. Real Hilbert bundles and (orthogonal) representations of discrete pmp groupoids on real Hilbert bundles can be defined in a similar fashion.

A representation $\pi$ of $G$ on $\mathcal{H}$ induces a representation $[[\pi]]$ of the inverse semigroup $[[G]]$ on $L^{2}\left(G^{0}, \mathcal{H}\right)$. This is defined by setting

$$
\left([[\pi]]_{\sigma} \xi\right)_{x}= \begin{cases}\pi_{x \sigma} \xi_{s(x \sigma)} & \text { if } x \in \sigma \sigma^{-1} \\ 0 & \text { otherwise. }\end{cases}
$$

In particular the restriction $[\pi]$ of $[[\pi]]$ to $[G]$ is a continuous representation of the Polish group $[G]$.

A unit section for $\mathcal{H}$ is a section $\xi$ such that $\left\|\xi_{x}\right\|=1$ for a.e. $x \in G^{0}$. A unit section $\xi$ for $\mathcal{H}$ is invariant if $\pi_{\gamma} \xi_{s(\gamma)}=\xi_{r(\gamma)}$ for a.e. $\gamma \in G$. We say that the representation $\pi$ of an ergodic discrete pmp groupoid $G$ on $\mathcal{H}$ is ergodic if it has no invariant unit sections. This is equivalent to the assertion that for some (equivalently, every) countable subgroup $\Gamma$ of $[G]$ that covers $G,\left.[\pi]\right|_{\Gamma}$ is ergodic. Let $\xi$ be a unit section for $\mathcal{H}$. We say that $\xi$ is cyclic if, for a.e. $x \in G^{0}$ one has that $\left\{\pi_{\gamma} \xi_{s(\gamma)}: \gamma \in x G\right\}$ has dense linear span in $\mathcal{H}_{x}$.

Let $\overline{\mathcal{H}}:=\bigsqcup_{x \in \mathcal{H}_{x}} \overline{\mathcal{H}}_{x}$, where $\overline{\mathcal{H}}_{x}$ denotes the conjugate Hilbert space of $\mathcal{H}_{x}$, with canonical conjugate linear isomorphism $\mathcal{H}_{x} \rightarrow \overline{\mathcal{H}}_{x}, \xi \mapsto \bar{\xi}_{x}$. The conjugate representation $\bar{\pi}$ of $G$ on $\overline{\mathcal{H}}$ is defined by $\bar{\pi}_{\gamma} \bar{\xi}=\overline{\pi_{\gamma} \xi}$ for $\gamma \in G$ and $\xi \in \mathcal{H}_{s(\gamma)}$.

Suppose that $\pi_{0}$ and $\pi_{1}$ are representations of $G$ on Hilbert bundles $\mathcal{H}_{0}$ and $\mathcal{H}_{1}$. Then one can consider the Hilbert bundle $\mathcal{H}_{0} \otimes \mathcal{H}_{1}:=\bigsqcup_{x \in G^{0}} \mathcal{H}_{0, x} \otimes \mathcal{H}_{1, x}$ and the representation $\pi_{0} \otimes \pi_{1}$ of $G$ on $\mathcal{H}_{0} \otimes \mathcal{H}_{1}$ defined in the obvious way.

Remark 2.1. In the following we will frequently use the following observation. Suppose that $G$ is an ergodic discrete pmp groupoid, $A \subset G^{0}$ is a non-null Borel set, and $\pi$ is a representation of $G$ on $\mathcal{H}$. Then one can consider the representation $\pi_{A}$ of $\left.G\right|_{A}$ on $\left.\mathcal{H}\right|_{A}=\bigsqcup_{x \in A} \mathcal{H}_{x}$ obtained from $\pi$ by restriction. If $\eta$ is an invariant section for $\left.\mathcal{H}\right|_{A}$, then there exists a unique invariant section $\xi$ for $G$ such that $\xi_{x}=\eta_{x}$ for $x \in A$. In particular, $\pi$ is ergodic if and only if $\pi_{A}$ is ergodic.

The notion of weak mixing representation has been introduced in [7, Definition 3.11]. The representation $\pi$ of an ergodic groupoid $G$ on $\mathcal{H}$ is weak mixing if, for every $\varepsilon>0, n \in \mathbb{N}$, and sections $\xi_{1}, \ldots, \xi_{n}$ for $\mathcal{H}$ there exists $t \in[G]$ such that, for every $i, j \in\{1,2, \ldots, n\}$,

$$
\int_{G^{0}}\left|\left\langle\xi_{j, x}, \pi_{x t} \xi_{i, s(x t)}\right\rangle\right| d \mu(x) \leq \varepsilon
$$

Several equivalent characterizations of such a notion have been established in [7, Subsection 3.3]. Particularly, a representation $\pi$ of an ergodic discrete pmp groupoid $G$ on $\mathcal{H}$ is weak mixing if and only if for some (equivalently, every) countable subgroup $\Gamma$ of $[G]$ that covers $G,\left.[\pi]\right|_{\Gamma}$ is weak mixing, if and only if $\mathcal{H}$ does not have an finite-dimensional invariant sub-bundle, if and only if $\pi \otimes \bar{\pi}$ is ergodic.

2.3. Property $(\mathbf{T})$. Suppose that $\Gamma$ is a countable discrete group, and $\pi$ is a representation of $\Gamma$ on a Hilbert space $\mathcal{H}$. If $F$ is a subset of $\Gamma$, and $\varepsilon>0$, then a unit vector $\xi$ of $\mathcal{H}$ is $(F, \varepsilon)$-invariant if it satisfies $\left\|\pi_{\gamma} \xi-\xi\right\| \leq \varepsilon$ for every $\gamma \in F$. The representation $\pi$ has almost invariant vectors if, for every finite subset $F$ of $\Gamma$ and for every $\varepsilon>0$, it has an $(F, \varepsilon)$-invariant vector. The group $\Gamma$ has property (T) if every representation of $\Gamma$ that has almost invariant unit vectors, it has an invariant unit vector [3]. A standard reference for the theory of property $(\mathrm{T})$ groups is [3].

The notion of property ( $\mathrm{T}$ ) for pmp equivalence relations has been introduced in [22]. A natural common generalization of the notion of property $(\mathrm{T})$ for discrete groups and pmp equivalence relations has been considered in [2]. Let $G$ be a discrete pmp groupoid with unit space $X$, and $\pi$ be a representation of $G$ on a Hilbert bundle $\mathcal{H}$. If $F$ is a subset of $[G]$ and $\varepsilon>0$, then we say that a unit section $\xi$ for $\mathcal{H}$ is $(F, \varepsilon)$-invariant if it satisfies $\left\|[\pi]_{t} \xi-\xi\right\| \leq \varepsilon$ in $L^{2}(X, \mathcal{H})$ for every $t \in F$. The representation $\pi$ has almost invariant unit sections if, for every finite subset $F$ of $[G]$ and $\varepsilon>0$, it has an $(F, \varepsilon)$-invariant unit section. The discrete pmp groupoid $G$ has property $(\mathrm{T})$ if every representation $\pi$ of $G$ that has almost invariant unit sections it has an invariant unit section [2, Definition 4.3].

The notion of property $(\mathrm{T})$ admits a natural relative version for subgroups. Suppose that $\Gamma$ is a countable discrete group, and $\Lambda \leq \Gamma$ is a subgroup. If $\pi$ is a representation of $\Gamma$ on a Hilbert space $\mathcal{H}$, 
then a unit vector $\xi$ in $\mathcal{H}$ is $\Lambda$-invariant if it is invariant for the restriction of $\pi$ to $\Lambda$. Then $\Lambda$ has relative property (T) in $\Gamma$, or the pair $\Lambda \leq \Gamma$ has property (T), if every representation of $\Gamma$ that has almost invariant unit vectors, it has a $\Lambda$-invariant unit vector. This notion admits a natural generalization to discrete pmp groupoids. Suppose that $G$ is a discrete pmp groupoid, $H$ is a subgroupoid of $G$, and $\pi$ is a unitary representation of $G$ on a Hilbert bundle $\mathcal{H}$. Then a unit section $\xi$ for $\mathcal{H}$ is $H$-invariant if it is invariant for the restriction of $\pi$ to $H$. Then $H$ has the relative property ( $\mathrm{T}$ ) in $G$, or pair $H \leq G$ has property (T), if every representation of $G$ that has almost invariant unit sections admits an $H$-invariant unit section. Clearly, when $H=G$, this recovers property (T) for a single discrete pmp groupoid.

A natural generalization of property $(\mathrm{T})$ from pairs of groups to triples of groups has been considered in [7, Definition 2.3]. Suppose that $\Gamma$ is a countable discrete group, and $\Delta \leq \Lambda \leq \Gamma$ are nested subgroups. Then the triple $\Delta \leq \Lambda \leq \Gamma$ has property (T) if every representation of $\Gamma$ with almost invariant $\Delta$-invariant unit vectors admits a $\Lambda$-invariant unit vector. Clearly, when $\Delta$ is the trivial subgroup one recovers the notion of property (T) for pairs of groups. Naturally, one can generalize such a notion to discrete pmp groupoids as follows. Suppose that $G$ is a discrete pmp groupoid, and $K \leq H \leq G$ are nested subgroupoids.

Definition 2.2. The triple $K \leq H \leq G$ has property (T) if, for every representation $\pi$ of $G$, if $\pi$ has almost invariant $K$-invariant unit sections, then $\pi$ has an $H$-invariant unit section.

Remark 2.3. When $H$ is ergodic, in Definition 2.2 one can equivalently require that every representation of $G$ with almost invariant $K$-invariant unit sections has a nonzero $H$-invariant unit section $\xi$. Indeed, in this case one has that there exists $\delta>0$ such that $\left\|\xi_{x}\right\|=\delta$ for a.e. $x \in H^{0}$. Therefore $\delta^{-1} \xi$ is a $H$-invariant unit section.

Again, when $K$ is the trivial subgroupoid of $H$-i.e. $K$ is equal to the common unit space of $H$ and $G$-one recovers the notion of property (T) for pairs $H \leq G$.

Several equivalent characterizations of property $(\mathrm{T})$ for pairs of groups are established in [11]. Furthermore, a cohomological characterization of property $(\mathrm{T})$ for discrete pmp groupoids is the main result of [2]. In this section we provide a characterization for triples of discrete pmp groupoids, subsuming the characterizations from $[2,11]$.

2.4. Cohomology of representations. Let $G$ be a discrete pmp groupoid, with subgroupoids $K \leq$ $H \leq G$. Denote by $X$ their common unit space. In the space of complex-valued Borel functions on $G$ consider the (Polish) topology generated by the pseudometrics

$$
d_{t}\left(\varphi, \varphi^{\prime}\right):=\int_{x \in X} \frac{\left|\varphi(t x)-\varphi^{\prime}(t x)\right|}{1+\left|\varphi(t x)-\varphi^{\prime}(t x)\right|} d \mu_{G^{0}}(x)
$$

where $t$ ranges within (a dense subset of) $[G]$. If $\mathcal{H}$ is a Hilbert bundle over $X$, then we let $S(G, \mathcal{H}$ ) be the space of Borel functions $G \rightarrow \mathcal{H}, \gamma \mapsto b_{\gamma} \in \mathcal{H}_{r(\gamma)}$ endowed with the topology generated by the pseudometrics

$$
d_{t}\left(b, b^{\prime}\right)=\int_{x \in X} \frac{\left\|b_{t x}-b_{t x}^{\prime}\right\|}{1+\left\|b_{t x}-b_{t x}^{\prime}\right\|} d \mu_{G^{0}}(x)
$$

Suppose that $\pi$ is a representation of $G$ on the Hilbert bundle $\mathcal{H}$. A cocycle for $\pi$ is an element $b$ of $S(G, \mathcal{H})$ such that $b_{\gamma_{1} \gamma_{2}}=b_{\gamma_{1}}+\pi_{\gamma_{1}}\left(b_{\gamma_{2}}\right)$ for a.e. $\left(\gamma_{1}, \gamma_{2}\right) \in G^{2}$. A cocycle $b$ for $\pi$ is $K$-trivial if $b_{\gamma}=0$ for a.e. $\gamma \in K$. A section $\xi$ for $\mathcal{H}$ defines a cocycle $c_{\pi}(\xi)$ for $\pi$ by setting $c_{\pi}(\xi)_{\gamma}=\xi_{r(\gamma)}-\pi_{\gamma} \xi_{s(\gamma)}$ for $\gamma \in G$. Cocycles of this form are called coboundaries. The section $\xi$ is $K$-invariant if and only if $c_{\pi}(\xi)$ is $K$-trivial. We denote the space of $K$-trivial cocycles for $\pi$ by $Z_{: K}^{1}(\pi)$, and the space of $K$-trivial coboundaries for $\pi$ by $B_{: K}^{1}(\pi)$. We let $Z_{: K, H}^{1}(\pi)$ be the set of restrictions to $H$ of elements of $Z_{: K}^{1}(\pi)$. The $K$-invariant $H$-relative cohomology group $H_{: K, H}^{1}(\pi)$ of $\pi$ is the quotient of $Z_{: K, H}^{1}(\pi)$ by the subgroup $B_{: K}^{1}\left(\left.\pi\right|_{H}\right)$. The same argument as in [2, Proposition 3.9] shows that $Z_{: K, H}^{1}(\pi)$ is a closed subset of $S(H, \mathcal{H})$.

The following result is established in [2, Theorem 3.19]. 
Theorem 2.4 (Anantharaman-Delaroche). Suppose that $G$ is an ergodic discrete pmp groupoid. Consider a representation $\pi$ of $G$ and a cocycle $b$ for $\pi$. The following assertions are equivalent:

(1) $\pi$ is a coboundary;

(2) there exists a non-null Borel subset $A$ of $G^{0}$ such that the function $A G A \rightarrow \mathbb{R}, \gamma \mapsto\left\|b_{\gamma}\right\|$ is bounded;

(3) there exists a non-null Borel subset $A$ of $G^{0}$ such that, for every $x \in A$, the function $A G x \rightarrow \mathbb{R}$, $\gamma \mapsto\left\|b_{\gamma}\right\|$ is bounded.

2.5. Functions of positive and negative type. Suppose that $G$ is a discrete pmp groupoid and $K \subset G$ is a subgroupoid. The following is a standard definition; see [19, Definition 4.1.2].

Definition 2.5. A complex-valued function $\varphi: G \rightarrow \mathbb{C}$ is of positive type if it is Borel, and for a.e. $x \in X$, for every $n \geq 1, \gamma_{1}, \ldots, \gamma_{n} \in x G$, and $\lambda_{1}, \ldots, \lambda_{n} \in \mathbb{C}$ one has that

$$
\sum_{i j} \bar{\lambda}_{i} \lambda_{j} \varphi\left(\gamma_{i}^{-1} \gamma_{j}\right) \geq 0
$$

A real-valued function $\varphi: G \rightarrow \mathbb{R}$ is of positive type if it is Borel, $\varphi(\gamma)=\varphi\left(\gamma^{-1}\right)$ for a.e. $\gamma \in G$, and for a.e. $x \in X$, for every $n \geq 1, \gamma_{1}, \ldots, \gamma_{n} \in x G$, and $\lambda_{1}, \ldots, \lambda_{n} \in \mathbb{R}$ one has that

$$
\sum_{i j} \bar{\lambda}_{i} \lambda_{j} \varphi\left(\gamma_{i}^{-1} \gamma_{j}\right) \geq 0
$$

The function $\varphi$ is $K$-invariant if $\varphi\left(\rho_{0} \gamma\right)=\varphi(\gamma)=\varphi\left(\gamma \rho_{1}\right)$ for every $\rho_{0}, \rho_{1} \in K$ and $\gamma \in G$ such that $\left(\rho_{0}, \gamma\right),\left(\gamma, \rho_{1}\right) \in G^{2}$. The function $\varphi$ is called normalized if $\varphi(x)=1$ for a.e. $x \in G^{0}$.

The same proof as [6, Proposition 5.3] gives the following.

Proposition 2.6. Suppose that $\varphi$ is a Borel complex-valued (respectively, real-valued) function on $G$. The following assertions are equivalent:

(1) $\varphi$ is a normalized $K$-invariant function of positive type;

(2) there exists a representation $\pi^{\varphi}$ of $G$ on a complex (respectively, real) Hilbert bundle $\mathcal{H}^{\varphi}$ and a K-invariant cyclic unit section $\xi^{\varphi}$ for $\mathcal{H}^{\varphi}$ such that $\varphi(\gamma)=\left\langle\xi_{r(\gamma)}, \pi_{\gamma} \xi_{s(\gamma)}\right\rangle$ for a.e. $\gamma \in G$.

The representation $\left(\pi^{\varphi}, \mathcal{H}^{\varphi}, \xi^{\varphi}\right)$ of $G$ is uniquely determined up to isomorphism, and it will called the GNS representation of $\varphi$.

The following definition is considered in [2, Proposition 5.19].

Definition 2.7. A real-valued function $\psi: G \rightarrow \mathbb{R}$ on $G$ is of conditionally negative type if it is Borel, $\psi\left(\gamma^{-1}\right)=\psi(\gamma)$ for a.e. $\gamma \in G, \psi(x)=0$ for a.e. $x \in G^{0}$, and

$$
\sum_{i, j=1}^{n} \bar{\lambda}_{i} \lambda_{j} \psi\left(\gamma_{i}^{-1} \gamma_{j}\right) \leq 0
$$

for a.e. $x \in G^{0}$, for every $n \geq 2, \lambda_{1}, \ldots, \lambda_{n} \in \mathbb{R}$ satisfying $\lambda_{1}+\cdots+\lambda_{n}=0$, and every $\gamma_{1}, \ldots, \gamma_{n} \in x G$.

A complex-valued function $\psi: G \rightarrow \mathbb{C}$ is of conditionally negative type if it satisfies the same properties where one consider complex scalars instead of real scalars.

The following proposition is essentially established in [2, Proposition 5.21].

Proposition 2.8. Suppose that $\psi$ is a Borel real-valued function on $G$. The following assertions are equivalent:

(1) $\psi$ is a $K$-invariant function of conditionally negative type;

(2) there exists a real Hilbert bundle $\mathcal{H}^{\psi}$, a representation $\pi^{\psi}$ of $G$ on $\mathcal{H}^{\psi}$, and a $K$-trivial cocycle $b^{\psi}$ for $\pi^{\psi}$ such that $\left\{b_{\gamma}^{\psi}: \gamma \in x G\right\}$ has dense linear span in $\mathcal{H}^{\psi}$, and such that $\psi(\gamma)=\left\|b_{\gamma}^{\psi}\right\|^{2}$ for a.e. $\gamma \in G$.

Furthermore one has that $\psi\left(\rho^{-1} \gamma\right)=\left\|b_{\gamma}^{\psi}-b_{\rho}^{\psi}\right\|^{2}$ for $\gamma, \rho \in G$ such that $r(\gamma)=r(\rho)$. 
The following two lemmas are consequences of [3, Theorem C.3.2] and [6, Proposition 5.18].

Lemma 2.9. Suppose that $\psi: G \rightarrow \mathbb{R}$ is a Borel real-valued function such that $\psi(x)=0$ for a.e. $x \in X$ and $\psi(\gamma)=\psi\left(\gamma^{-1}\right)$ for a.e. $\gamma \in G$. Then the following assertions are equivalent:

(1) $\psi$ is conditionally of negative type;

(2) the function $\gamma \mapsto \exp (-t \psi(\gamma))$ is of positive type for every $t>0$.

Lemma 2.10. Suppose that $\psi: G \rightarrow \mathbb{C}$ is a complex-valued function of conditionally negative type. Then $\operatorname{Re}(\psi)$ is a real-valued function of conditionally positive type. Furthermore, $\psi$ is bounded if and only if $\operatorname{Re}(\psi)$ is bounded and, for every $x \in G^{0},\left.\operatorname{Re}(\psi)\right|_{G x}$ is bounded if and only if $\left.\psi\right|_{G x}$ is bounded.

2.6. A cohomological characterization. We now provide a characterization of property ( $\mathrm{T}$ ) for triples of groupoids, including in particular a cohomological characterization; see Theorem 2.13 below. Such a cohomological characterization generalizes the one in [2] for single groupoids. Even in this case, some parts of the proof presented here are different, and in fact closer in spirit to the group case as in [11].

Lemma 2.11. Suppose that $G$ is a discrete pmp groupoid with unit space $(X, \mu)$, and $H$ is an ergodic subgroupoid of $G$. Let $\psi: G \rightarrow \mathbb{R}$ be a function of conditionally negative type. For $t>0$ let $\pi^{(t)}$ be the representation on the Hilbert bundle $\mathcal{H}^{(t)}$ and $\xi^{(t)}$ be the section of $\mathcal{H}^{(t)}$ obtained from the function of positive type $\exp (-t \psi)$ via the GNS construction. The following assertions are equivalent:

(1) there exists a non-null Borel subset $A$ of $X$ such that, for a.e. $x \in A,\left.\psi\right|_{A H x}$ is bounded;

(2) for every $t>0,\left.\pi^{(t)}\right|_{H}$ is not ergodic;

(3) there exists $t>0$ such that $\left.\pi^{(t)}\right|_{H}$ is not weak mixing;

(4) for every non-null Borel subset $B$ of $X$ there exists a non-null Borel subset $A$ of $X$ contained in $B$ such that, for a.e. $x \in A,\left.\psi\right|_{A H x}$ is bounded;

(5) for every non-null Borel subset $B$ of $X$, there exists a non-null Borel subset $A$ of $X$ contained in $B$ such that $\left.\psi\right|_{A H A}$ is bounded.

Proof. (1) $\Rightarrow(2)$ Suppose that $\left.\psi\right|_{A H x}$ is bounded by $c_{x}$ for a.e. $x \in A$, where $A$ is a non-null Borel subset of $X$. In view of Remark 2.1, after replacing $G$ with $\left.G\right|_{A}$, we can assume that $A=X$. Set $c(t):=\int \exp \left(-t c_{x}\right) d \mu(x)$ for $t>0$. Define $C$ to be the closed convex hull of $\left\{\left[\pi^{(t)}\right] \xi^{(t)}: \sigma \in[H]\right\}$. We claim that $\|\xi\| \geq c(t)$ for every $\xi \in C$. It is enough to consider the case when $\xi=\sum_{i=1}^{n} s_{i}\left[\pi^{(t)}\right]_{\sigma_{i}} \xi^{(t)}$ for $\sigma_{i} \in[H]$ and $s_{i} \in[0,1]$ such that $s_{1}+\cdots+s_{n}=1$. In this case, we have that

$$
\begin{aligned}
\left\|\sum_{i} s_{i}\left[\pi^{(t)}\right]_{\sigma_{i}} \xi^{(t)}\right\|^{2} & =\sum_{i j} s_{i} s_{j}\left\langle\xi^{(t)},\left[\pi^{(t)}\right]_{\sigma_{i}^{-1} \sigma_{j}} \xi^{(t)}\right\rangle d \mu(x) \\
& =\sum_{i j} s_{i} s_{j} \int \exp \left(-t \psi\left(\sigma_{i}^{-1} \sigma_{j} x\right)\right) d \mu(x) \\
& \geq \sum_{i j} s_{i} s_{j} \int \exp \left(-t c_{x}\right) d \mu(x) \\
& =c(t) .
\end{aligned}
$$

Pick now an element $\xi$ of $C$ of minimal norm. Observe that $\xi$ is nonzero, and it is $H$-invariant by uniqueness.

$(2) \Rightarrow(3)$ Obvious.

$(3) \Rightarrow(1)$ Suppose that (1) does not hold. Thus for every non-null Borel subset $A$ of $X$, there exists a non-null Borel subset $B$ such that for every $x \in B,\left.\psi\right|_{A H x}$ is unbounded. Fix $t>0$. We claim that this implies that, for any unit section $\eta$ for $\mathcal{H}^{(t)} \otimes \overline{\mathcal{H}}^{(t)}$ and $\varepsilon \in(0,1)$ there exists $\rho \in[H]$ such that one has that

$$
\|\|\left[\pi^{(t)} \otimes \bar{\pi}^{(t)}\right]_{\rho} \eta-\eta \|^{2}-2 \mid<\varepsilon
$$


In particular this shows that $\left.\left(\pi^{(t)} \otimes \bar{\pi}^{(t)}\right)\right|_{H}$ is ergodic, and hence $\left.\pi^{(t)}\right|_{H}$ is weak mixing. Since $\xi^{(t)}$ is a cyclic unit section for $\mathcal{H}^{(t)}$, it suffices to prove the claim when $\eta$ is of the form

$$
x \mapsto \sum_{i j=1}^{n} a_{i j}(x)\left(\left[\pi^{(t)}\right]_{\sigma_{i}} \xi^{(t)}\right)_{x} \otimes\left(\left[\bar{\pi}^{(t)}\right]_{\sigma_{j}^{\prime}} \bar{\xi}^{(t)}\right)_{x},
$$

where $n \in \mathbb{N}, a_{i j} \in L^{\infty}(X)$, and $\sigma_{i}, \sigma_{j}^{\prime} \in[G]$ for $i, j=1,2, \ldots, n$. For $z \in X$, fix $M(z)>0$ such that

$$
\max \left\{\left|a_{i j}\left(r\left(\sigma_{k}^{-1} z\right)\right)\right| \exp (-t M(z)): 1 \leq i, j, k \leq n\right\} \leq \frac{\varepsilon}{n^{4}} .
$$

By assumption for every non-null Borel subset $A$ of $X$ there exists a non-null Borel subset $B$ of $A$ such that, for every $x \in B,\left.\psi\right|_{A H x}$ is unbounded. This easily implies that there exists $\rho \in[H]$ such that, for a.e. $z, w \in X$, for every $1 \leq i, k \leq n$,

$$
\psi(z \rho w) \geq M(z)^{1 / 2}+\psi\left(\sigma_{k} z\right)^{1 / 2}+\psi\left(w \sigma_{i}^{\prime}\right)^{1 / 2} .
$$

We have that

$$
\begin{aligned}
& \frac{1}{2}\left|\left\|\left([\pi]_{\rho} \otimes[\bar{\pi}]_{\rho}\right) \eta-\eta\right\|^{2}-2\right| \\
= & \operatorname{Re} \sum_{i, j, k, l=1}^{n} \int_{x \in X} \bar{a}_{k l}(x) a_{i j}(x)\left\langle\xi_{x}^{(t)} \otimes \bar{\xi}_{x}^{(t)}, \pi_{x \sigma_{k}^{-1} \rho \sigma_{i}^{\prime}}^{(t)} \xi_{s\left(x \sigma_{k}^{-1} \rho \sigma_{i}^{\prime}\right)}^{(t)} \otimes \bar{\pi}_{x \sigma_{l}^{-1} \rho \sigma_{j}^{\prime}}^{(t)} \bar{\xi}_{s\left(x \sigma_{l}^{-1} \rho \sigma_{j}^{\prime}\right)}^{(t)}\right\rangle d \mu(x) \\
= & \operatorname{Re} \sum_{i, j, k, l=1}^{n} \int_{x \in X} \bar{a}_{k l}(x) a_{i j}(x) \exp \left(-t \psi\left(x \sigma_{k}^{-1} \rho \sigma_{i}^{\prime}\right)-t \psi\left(x \sigma_{l}^{-1} \rho \sigma_{j}^{\prime}\right)\right) d \mu(x) .
\end{aligned}
$$

Now let $\left(\mathcal{H}^{\psi}, b^{\psi}, \pi^{\psi}\right)$ be a triple obtained from $\psi$ as in Proposition 2.8. Thus we have that $\mathcal{H}^{\psi}$ is a real Hilbert bundle over $X, \pi^{\psi}$ is a representation of $G$ on $\mathcal{H}^{\psi}$, and $b^{\psi}$ is a cocycle for $\pi^{\psi}$ such that

$$
\psi\left(g^{-1} h\right)=\left\|b_{g}^{\psi}-b_{h}^{\psi}\right\|^{2}
$$

for $g, h \in G$. Thus, for a.e. $x \in X$, by the choice of $\rho$,

$$
\begin{aligned}
\psi\left(x \sigma_{k}^{-1} \rho \sigma_{i}^{\prime}\right) & =\left\|b_{r\left(\sigma_{k} x\right) \rho \sigma_{i}^{\prime}}-b_{\sigma_{k} x}\right\|^{2} \\
& =\left\|b_{r\left(\sigma_{k} x\right) \rho}+\pi_{r\left(\sigma_{k} x\right) \rho} b_{s\left(r\left(\sigma_{k} x\right) \rho\right) \sigma_{i}^{\prime}}-b_{\sigma_{k} x}\right\|^{2} \\
& \geq\left(\left\|b_{r\left(\sigma_{k} x\right) \rho}\right\|-\left\|b_{s\left(r\left(\sigma_{k} x\right) \rho\right) \sigma_{i}^{\prime}}\right\|-\left\|b_{\sigma_{k} x}\right\|\right)^{2} \\
& =\left(\psi\left(r\left(\sigma_{k} x\right) \rho\right)^{1 / 2}-\psi\left(s(\rho) \sigma_{i}^{\prime}\right)^{1 / 2}-\psi\left(\sigma_{k} x\right)^{1 / 2}\right)^{2} \\
& \geq M\left(r\left(\sigma_{k} x\right)\right),
\end{aligned}
$$

where we used the choice of $\rho$ at the last step. Similarly,

$$
\psi\left(\gamma_{l}^{-1} \rho \gamma_{j}^{\prime}\right) \geq M\left(r\left(\sigma_{l} x\right)\right) .
$$

Hence we have that

$$
\begin{aligned}
& \left|\left\|\left([\pi]_{\rho} \otimes[\bar{\pi}]_{\rho}\right) \eta-\eta\right\|^{2}-2\right| \\
= & \operatorname{Re} \sum_{i, j, k, l=1}^{n} \int_{x \in X} \bar{a}_{k l}(x) a_{i j}(x) \exp \left(-t \psi\left(x \sigma_{k}^{-1} \rho \sigma_{i}^{\prime}\right)-t \psi\left(x \sigma_{l}^{-1} \rho \sigma_{j}^{\prime}\right)\right) d \mu(x) \\
\leq & \operatorname{Re} \sum_{i, j, k, l=1}^{n} \int_{x \in X} \bar{a}_{k l}(x) a_{i j}(x) \exp \left(-t M\left(r\left(\sigma_{k} x\right)\right)-t M\left(r\left(\sigma_{l} x\right)\right)\right) d \mu(x) \leq \varepsilon
\end{aligned}
$$

by ((i)). This concludes the proof. 
$(1) \Leftrightarrow(4)$ Suppose that $B$ is a non-null Borel subset of $X$, and $\pi$ is a representation of $G$. Since $H$ is ergodic, by Remark 2.1 the equivalence $(1) \Leftrightarrow(4)$ follows from the equivalence $(1) \Leftrightarrow(2)$ after replacing $G$ with $\left.G\right|_{B}$.

$(4) \Leftrightarrow(5)$ This follows from Theorem 2.4.

Lemma 2.12. Let $\pi$ be a representation of a discrete pmp groupoid $G$ on $\mathcal{H}$ and fix $\delta>0$. Suppose that $\xi$ is a unit section for $\mathcal{H}$. Assume that for every $\gamma \in G$ one has that $\left\|\xi_{r(\gamma)}-\pi_{\gamma} \xi_{s(\gamma)}\right\| \leq \delta$. Then there exists an invariant section $\eta$ of $\mathcal{H}$ such that $\left\|\eta_{x}-\xi_{x}\right\| \leq \delta$ for a.e. $x \in X$.

Proof. For every $x \in G^{0}$ let $C_{x}$ be the closed convex hull of $\left\{\pi_{\gamma^{-1}} \xi_{r(\gamma)}: \gamma \in G x\right\}$. Let then $\eta_{x}$ be the (unique) element of minimal norm of $C_{x}$ for $x \in G^{0}$. If $x \in X$ then we have that, for every $\gamma_{1}, \ldots, \gamma_{n} \in G x$ and $s_{1}, \ldots, s_{n} \in[0,1]$ such that $s_{1}+\cdots+s_{n}=1$ one has that

$$
\left\|\sum_{i} s_{i} \pi_{\gamma_{i}^{-1}} \xi_{r\left(\gamma_{i}\right)}-\xi_{x}\right\| \leq \sum_{i} s_{i}\left\|\xi_{r\left(\gamma_{i}\right)}-\pi_{\gamma_{i}} \xi_{x}\right\| \leq \delta .
$$

Therefore

$$
\left\|\zeta-\xi_{x}\right\| \leq \delta
$$

for every $\zeta \in C_{x}$ and in particular

$$
\left\|\eta_{x}-\xi_{x}\right\| \leq \delta .
$$

By uniqueness of the element of least norm in $C_{x}$ one also has that $\eta$ is invariant.

The proof of the following result is inspired by [11, Theorem 1.2] and [2, Theorem 4.8 and Theorem 4.12].

Theorem 2.13. Let $G$ be a discrete pmp groupoid, and $K \leq H \leq G$ be subgroupoids. Fix a countable subgroup $\Gamma$ of $[G]$ that covers $G$. Let $(X, \mu)$ be the common unit space of $K, H, G$. Suppose that $H$ is ergodic. The following statements are equivalent:

(1) There exists a finite subset $F$ of $[G]$ and $\delta>0$ such that, whenever a representation $\pi$ of $G$ has an $(F, \delta)$-invariant $K$-invariant unit section, then $\pi$ has an $H$-invariant unit section;

(2) There exists a finite subset $F$ of $[G]$ and $\delta>0$ such that, whenever a representation $\pi$ of $G$ on a Hilbert bundle $\mathcal{H}$ has an $(F, \delta)$-invariant $K$-invariant unit section, then $\mathcal{H}$ contains a finite-dimensional $\left.\pi\right|_{H}$-invariant sub-bundle;

(3) For every complex-valued $K$-invariant Borel function $\psi$ on $G$ which is conditionally of negative type, there exists a non-null Borel set $A$ of $X$ such that, for every $x \in A,\left.\psi\right|_{A H x}$ is bounded;

(4) For any representation $\pi$ of $G$, one has that $H_{: K, H}^{1}(\pi)$ is trivial.

(5) the triple $K \leq H \leq G$ has property (T);

(6) For every $\varepsilon>0$ and non-null Borel subset $B$ of $X$ there exists a finite subset $F$ of $\Gamma$ and $\delta>0$ such that for every normalized $K$-invariant function of positive type $\varphi$ on $G$ such that $\max _{t \in F} \int_{x \in X}|\varphi(x t)-1|^{2} d \mu(x) \leq \delta$, there is a non-null Borel subset $A$ of $B$ such that $\operatorname{Re}(1-\varphi(\gamma)) \leq \varepsilon$ for a.e. $\gamma \in A H A$;

(7) For every $\varepsilon>0$ and non-null Borel subset $B$ of $X$ there exists a finite subset $F$ of $\Gamma$ and $\delta>0$ such that, if $\pi$ is a representation of $G$ on a Hilbert bundle $\mathcal{H}$, and $\xi$ is a $(F, \delta)$-invariant $K$ invariant unit section for $\mathcal{H}$, then there is a non-null Borel subset $A$ of $B$ and an $H$-invariant section $\eta$ for $\pi$ such that $\left\|\xi_{x}-\eta_{x}\right\| \leq \varepsilon$ for a.e. $x \in A$;

(8) For every $\varepsilon>0$, there exists a finite subset $F$ of $[G]$ and $\delta>0$ such that for every normalized $K$-invariant function of positive type $\varphi$ on $G$ such that $\max _{t \in F} \int_{x \in X}|\varphi(x t)-1|^{2} d \mu(x) \leq \delta$, there is a non-null Borel subset $A$ of $X$ such that $\operatorname{Re}(1-\varphi(\gamma)) \leq \varepsilon$ for a.e. $\gamma \in A H A$;

(9) For every $\varepsilon>0$, there exists a finite subset $F$ of $[G]$ and $\delta>0$ such that, if $\pi$ is a representation of $G$ on a Hilbert bundle $\mathcal{H}$, and $\xi$ is a $(F, \delta)$-invariant $K$-invariant unit section for $\mathcal{H}$, then there is a non-null Borel subset $A$ of $X$ and an $H$-invariant section $\eta$ for $\pi$ such that $\left\|\xi_{x}-\eta_{x}\right\| \leq \varepsilon$ for a.e. $x \in A$. 
Proof. Fix an increasing sequence $\left(F_{n}\right)$ of finite subsets of $[G]$ whose union is $\Gamma$.

$(1) \Rightarrow(2)$ Obvious.

$(2) \Rightarrow(3)$ This is a consequence of Lemma 2.11 .

$(3) \Rightarrow(4)$ As in the proof of [2, Proposition 4.13], it is enough to consider the case when $\pi$ is a representation of $G$ on a bundle of real Hilbert spaces; see also [2, Lemma 4.11] and [6, page 49]. Suppose that $b$ is a $K$-trivial cocycle for $\pi$. Define the $K$-invariant function of conditional negative type $\psi: G \rightarrow \mathbb{R}$ by $\psi(\gamma)=\left\|b_{\gamma}\right\|^{2}$. Then by assumption, there exists a non-null Borel subset $A$ of $X$ such that, for every $x \in A,\left.\psi\right|_{A H x}$ is bounded. This implies by Theorem 2.4 that the restriction of $b$ to $H$ is a coboundary for $\left.\pi\right|_{H}$. Thus $H_{: K, H}^{1}(\pi)$ is trivial.

$(4) \Rightarrow(5)$ Suppose by contradiction that there exists a representation $\pi$ of $G$ that has almost invariant $K$-invariant unit sections but it does not have an $H$-invariant unit section. The hypothesis implies that $B_{: K}^{1}\left(\left.\pi\right|_{H}\right)=Z_{: K, H}^{1}(\pi)$. In particular, $B_{: K}^{1}\left(\left.\pi\right|_{H}\right)$ is a closed subspace of $S(H, \mathcal{H})$. Let $S_{: K}(X, \mathcal{H})$ be the space of $K$-invariant unit sections for $\mathcal{H}$, which is a closed subspace of the space $S(X, \mathcal{H})$ of sections for $\mathcal{H}$. Define a map $\beta$ from the space $S_{: K}(X, \mathcal{H})$ to $B_{: K}^{1}\left(\left.\pi\right|_{H}\right)$ by

$$
\beta(\xi)_{\gamma}:=\xi_{r(\gamma)}-\pi_{\gamma} \xi_{s(\gamma)}
$$

for $\xi \in S(X, \mathcal{H})$ and $\gamma \in H$. This map is a continuous linear map from $S_{: K}(X, \mathcal{H})$ onto $B_{: K}^{1}\left(\left.\pi\right|_{H}\right)$. Since by assumption $\pi$ does not have an $H$-invariant unit section, such a map is injective. Since a continuous linear isomorphism between metrizable complete topological vector spaces is a homeomorphism, $\beta$ is a homeomorphism. Since by assumption $\pi$ has almost invariant $K$-invariant unit sections, we can find a sequence $\left(\xi^{(n)}\right)$ of $K$-invariant unit sections in $S_{: K}(X, \mathcal{H})$ such that $\beta\left(\xi^{(n)}\right) \rightarrow 0$. Therefore $\xi^{(n)} \rightarrow 0$, contradicting the fact that the $\xi^{(n)}$ 's are unit sections.

$(5) \Rightarrow(1)$ Assume that (1) does not hold. Then for every $n \in \mathbb{N}$ there exists a representation $\pi^{(n)}$ on $\mathcal{H}^{(n)}$ without $H$-invariant unit sections which has a $\left(F_{n}, 2^{-n}\right)$-invariant $K$-invariant unit section $\xi^{(n)}$. One can then consider the direct sum $\pi$ of $\pi^{(n)}$ for $n \in \mathbb{N}$. Then $\pi$ has almost invariant $K$-invariant unit sections. Hence by assumption it has an $H$-invariant unit section $\xi$. One can write $\xi$ as the direct sum of sections $\xi^{(n)}$ for $\mathcal{H}^{(n)}$ for $n \in \mathbb{N}$. Since $\xi$ is a $H$-invariant, one has that $\xi^{(n)}$ is $H$-invariant for every $n \in \mathbb{N}$. Since $\xi$ is a unit section, there exists $n \in \mathbb{N}$ such that $\xi^{(n)}$ is not identically zero. Since $H$ is ergodic, this contradicts the assumption that $\pi^{(n)}$ does not have $H$-invariant unit sections.

$(3) \Rightarrow(6)$ Suppose that (6) fails. Then there exists $c>0$ and a non-null Borel subset $B$ of $X$ such that for every $n \in \mathbb{N}$ one can find a $K$-invariant function of positive type $\varphi$ on $G$ such that $\max _{t \in F_{n}} \int_{x \in X}|\varphi(x t)-1| d \mu(x) \leq 2^{-n}$ and for every non-null Borel subset $A$ of $B$ the set of $\gamma \in A H A$ such that $\operatorname{Re}(1-\varphi(\gamma)) \geq c$ is non-null. This allows one to find a sequence $\left(\varphi_{n}\right)$ of $K$-invariant functions of positive type on $G$ and Borel subsets $X_{n}$ of $X$ such that $\mu\left(X_{n}\right) \geq 1-2^{-n},|\varphi(x t)-1| \leq$ $2^{-n}$ for $x \in X_{n}$ and $t \in F_{n}$, and such that for every non-null Borel subset $A$ of $B$ the set of $\gamma \in A H A$ such that $\operatorname{Re}(1-\varphi(\gamma)) \geq c$ is non-null. One can then define, for a.e. $\gamma \in G$,

$$
\psi(\gamma)=\sum_{n \in \mathbb{N}} 2^{n} \operatorname{Re}\left(1-\varphi_{n}(\gamma)\right)
$$

This gives a $K$-invariant function of conditionally negative type on $G$ such that $\left.\psi\right|_{A H A}$ is unbounded for every non-null Borel subset $A$ of $B$. By Lemma 2.11, this implies that, for every non-null Borel subset $A$ of $X$, for a.e. $x \in A,\left.\psi\right|_{A H x}$ is unbounded. Thus $\psi$ contradicts (3).

$(6) \Rightarrow(7)$ Fix $\varepsilon>0$ and a non-null Borel subset $B$ of $X$. By assumption there exist a finite subset $F$ of $\Gamma$ and $\delta>0$ such that, for every $K$-invariant normalized function of positive type $\varphi$ on $G$ such that $\max _{t \in F} \int_{x \in X}|\varphi(x t)-1| d \mu(x) \leq \delta$, there is a non-null Borel subset $A$ of $B$ such that $\operatorname{Re}(1-\varphi(\gamma)) \leq \varepsilon$ for every $\gamma \in A H A$. Suppose that $\pi$ is a representation of $G$ on $\mathcal{H}$ that has a $K$-invariant unit section $\xi$ satisfying $\left\|[\pi]_{t} \xi-\xi\right\| \leq \delta$ for $t \in F$. Define the $K$-invariant normalized function of positive type $\varphi$ on $G$ by $\varphi(\gamma)=\left\langle\xi_{r(\gamma)}, \pi_{\gamma} \xi_{s(\gamma)}\right\rangle$. Then we have that, for $t \in F$,

$$
\begin{aligned}
\int_{x \in X}|\varphi(x t)-1| d \mu(x) & =\int_{x \in X}\left|\left\langle\xi_{x}, \pi_{x t} \xi_{s(x t)}\right\rangle-1\right| d \mu(x)=\int_{x \in X}\left|\left\langle\xi_{x}, \pi_{x t} \xi_{s(x t)}-\xi_{x}\right\rangle\right| d \mu(x) \\
& =\left|\left\langle\xi,[\pi]_{t} \xi-\xi\right\rangle\right| \leq\left\|[\pi]_{t} \xi-\xi\right\| \leq \delta .
\end{aligned}
$$


Therefore by assumption there exists a non-null Borel subset $A$ of $B$ such that, for $\gamma \in A H A$, one has that $\operatorname{Re}(1-\varphi(\gamma)) \leq \varepsilon$. Therefore we have that, for $\gamma \in A H A$,

$$
\frac{1}{2}\left\|\pi_{\gamma} \xi_{s(\gamma)}-\xi_{r(\gamma)}\right\|^{2}=\operatorname{Re}(1-\varphi(\gamma)) \leq \varepsilon
$$

Therefore by Lemma 2.12 applied to the representation $\pi_{A}$ of $\left.H\right|_{A}$ on $\left.\mathcal{H}\right|_{A}$ obtained from $\pi$ by restriction, we have that there exists a unit section $\eta$ for $\left.\mathcal{H}\right|_{A}$ which is invariant for $\pi_{A}$ and such that $\left\|\xi_{x}-\eta_{x}\right\| \leq \varepsilon$ for $x \in A$. Since $H$ is ergodic, this concludes the proof by Remark 2.1.

$(8) \Rightarrow(9)$ This is the same as $(6) \Rightarrow(7)$.

Finally the implications $(7) \Rightarrow(1),(6) \Rightarrow(8)$, and $(9) \Rightarrow(1)$ are obvious.

Remark 2.14. Theorem 2.13 in the case when $G$ is a countable discrete group and $K$ is the trivial subgroup recovers [11, Theorem 1]. Theorem 2.13 recovers [2, Theorems 4.8, Theorem 4.12 and Theorem 5.22] in the case when $H=G$ and $K$ is the trivial subgroupoid.

2.7. Property $(\mathbf{T})$ for action groupoids. Suppose that $(X, \mu)$ is a standard probability space. A standard probability space fibered over $(X, \mu)$ is a triple $(Y, \nu, p)$ where $(Y, \nu)$ is a standard probability space, and $p: Z \rightarrow X$ is a Borel map such that $p_{*} \nu=\mu$. We also write $(Y, \nu)=\bigsqcup_{x \in X}\left(Y_{x}, \nu_{x}\right)$ where $\left(\nu_{x}\right)_{x \in X}$ is the disintegration of $\nu$ with respect to $\mu$. One can consider the space Aut $\left(\bigsqcup_{x \in X} Y_{x}\right)$ of Borel maps $T: Y_{x} \rightarrow Y_{y}$ for $x, y \in X$ such that $T_{*} \nu_{x}=\nu_{y}$. One can define a standard Borel structure on Aut $\left(\bigsqcup_{x \in X} Y_{x}\right)$ that turns it into a standard Borel groupoid, whose unit space can be identified with $X$.

Suppose that $G$ is a discrete pmp groupoid, and $\bigsqcup_{x \in X} Y_{x}$ is a standard probability space fibered over $G^{0}$. A probability-measure-preserving (pmp) action $\theta$ of $G$ on $\bigsqcup_{x \in X} Y_{x}$ is a homomorphism $\gamma \mapsto \theta_{\gamma}$ from $G$ to Aut $\left(\bigsqcup_{x \in X} Y_{x}\right)$ that is the identity on the unit space. We set $\gamma \cdot{ }^{\theta} y=\theta_{\gamma}(y)$ for $\gamma \in G$ and $y \in Y_{s(\gamma)}$. One can then define the transformation groupoid $G \ltimes^{\theta} Y$. This is the set of pairs $(\gamma, y)$ such that $\gamma \in G$ and $y \in Y_{s(\gamma)}$, which is a Borel subset of $G \times Y$ endowed with the product topology. Identifying an element $y$ of $Y_{x}$ for $x \in X$ with the pair $(x, y)$, one can identify $Y$ with the unit space of $G \ltimes^{\theta} Y$. The source and range maps on $G \ltimes^{\theta} Y$ are defined by $s(\gamma, y)=y$ and $r(\gamma, y)=\gamma \cdot{ }^{\theta} y$. Composition of arrows is given by $(\gamma, y)\left(\gamma^{\prime}, y^{\prime}\right)=\left(\gamma \gamma^{\prime}, y^{\prime}\right)$ whenever $\gamma^{\prime} \cdot{ }^{\theta} y^{\prime}=y$.

Suppose that $G$ is a discrete pmp groupoid, and $\theta$ is an action of $G$ on the standard probability space $Y=\bigsqcup_{x \in G^{0}} Y_{x}$. One can then consider the transformation groupoid $G \ltimes^{\theta} Y$. Suppose that $K \leq H \leq G$ are subgroupoids. A representation $\pi$ of $G$ on $\mathcal{H}$ induces a representation $\pi \ltimes^{\theta} Y$ of $G \ltimes^{\theta} Y$ on $\mathcal{H}$ defined by $\left(\pi \ltimes^{\theta} Y\right)_{\gamma \ltimes^{\theta} x}(\xi)=\pi_{\gamma}(\xi)$ for $\xi \in \mathcal{H}_{x}$. Let $b \in S(G, \mathcal{H})$ be a cocycle for $\pi$. Then one can define a cocycle $b \ltimes^{\theta} Y$ for $\pi \ltimes^{\theta} Y$ by setting $\left(b \ltimes^{\theta} Y\right)_{\gamma{ }^{\theta} x}=b_{\gamma}$. It is clear that if $b$ is $K$-trivial then $b \ltimes^{\theta} Y$ is $K \ltimes^{\theta} Y$-trivial. Furthermore if $\left.b\right|_{H}$ is a coboundary for $\left.\pi\right|_{H}$, then $\left.b\right|_{H} \ltimes^{\theta} Y$ is a coboundary for $\left.\left(\pi \ltimes^{\theta} Y\right)\right|_{H \ltimes^{\theta} Y}$. Therefore the assignment $b \mapsto b \ltimes^{\theta} Y$ defines a homomorphism $H_{: K, H}^{1}(\pi) \rightarrow H_{: K \ltimes^{\theta} Y, H \ltimes{ }^{\theta} Y}^{1}(\pi \ltimes Y)$. The following lemma is an immediate consequence of [2, Lemma $5.12]$.

Lemma 2.15. Adopting the notation above, suppose that $H$ is ergodic and the action $\left.\theta\right|_{H}$ of $H$ on $Y$ is ergodic. Then the homomorphism $H_{: K, H}^{1}(\pi) \rightarrow H_{: K \ltimes^{\theta} Y, H \ltimes{ }^{\theta} Y}^{1}(\pi \ltimes Y)$ is injective.

Proof. Suppose that $b$ is a $K$-invariant cocycle for $\pi$ on $\mathcal{H}$. Assume that $\left.\left(b \ltimes^{\theta} Y\right)\right|_{H^{\theta} Y}$ is a coboundary. Then by [2, Lemma 5.12], $\left.b\right|_{H}$ is a coboundary. Thus the map $H_{: K, H}^{1}(\pi) \rightarrow H_{: K \ltimes^{\theta} Y, H \ltimes{ }^{\theta} Y}^{1}(\pi \ltimes Y)$ is injective.

Suppose now, adopting the notation above, that $\pi$ is a representation of $H \ltimes^{\theta} Y$ on $\mathcal{H}$. One can then define the induced representation $\hat{\pi}$ of $G$ as follows. Consider the Hilbert bundle $\widehat{\mathcal{H}}=$ $\bigsqcup_{x \in X} L^{2}\left(Y_{x}\right) \otimes \mathcal{H}_{x} \cong \bigsqcup_{x \in X} L^{2}\left(Y_{x}, \mathcal{H}_{x}\right)$. Then the representation $\hat{\pi}$ on $\widehat{\mathcal{H}}$ is defined by setting, for $\gamma \in G$ and $\xi \in L^{2}\left(Y_{s(\gamma)}, \mathcal{H}_{s(\gamma)}\right), \hat{\pi}_{\gamma} \xi$ to be the element of $L^{2}\left(Y_{r(\gamma)}, \mathcal{H}_{r(\gamma)}\right)$ given by

$$
\left(\hat{\pi}_{\gamma} \xi\right)(y)=\pi_{\gamma \ltimes^{\theta} \gamma^{-1} y} \xi\left(\gamma^{-1} \cdot \theta y\right)
$$


for $y \in Y_{r(\gamma)}$. Observe that this is indeed a representation. In fact we have that

$$
\begin{aligned}
\hat{\pi}_{\gamma}\left(\hat{\pi}_{\rho} \xi\right) & =\pi_{\gamma \ltimes^{\theta} \gamma^{-1} y}\left(\hat{\pi}_{\rho} \xi\right)\left(\gamma^{-1} \cdot \theta y\right)=\pi_{\gamma \ltimes^{\theta} \gamma^{-1} y}\left(\pi_{\rho \rtimes \theta} \rho^{-1} \gamma^{-1} y\right) \xi\left(\rho^{-1} \gamma^{-1} \cdot{ }^{\theta} y\right) \\
& =\pi_{\gamma \rho \ltimes^{\theta}(\gamma \rho)^{-1} y} \xi\left((\gamma \rho)^{-1} \cdot{ }^{\theta} y\right)=\hat{\pi}_{\gamma \rho}(y) .
\end{aligned}
$$

Given a section $\xi$ for $\mathcal{H}$ one can define the section $\hat{\xi}$ of $\widehat{\mathcal{H}}$ by setting $\hat{\xi}_{x}=\left.\xi\right|_{Y_{x}} \in L^{2}\left(Y_{x}, \mathcal{H}_{x}\right)$ for $x \in G^{0}$. It is clear that if $\xi$ is $K \ltimes Y$-invariant, then $\hat{\xi}$ is $K$-invariant. Furthermore it is shown in [2, Section 5] that if $\left(\xi_{n}\right)$ is a sequence of almost $\pi$-invariant unit sections for $\mathcal{H}$, then $\left(\hat{\xi}_{n}\right)$ is a sequence of almost $\hat{\pi}$-invariant unit sections for $\widehat{\mathcal{H}}$. As in the proof of [2, Theorem 5.15] one can deduce from Lemma 2.15 and these observations the following.

Theorem 2.16. Suppose that $G$ is a discrete pmp groupoid, and $K \leq H \leq G$ are subgroupods such that $H$ is ergodic. Let $\theta$ be an action of $G$ on a standard probability space $Y$ such that $\left.\theta\right|_{H}$ is ergodic. Then $K \leq H \leq G$ has property (T) if and only if $K \ltimes^{\theta} Y \leq H \ltimes^{\theta} Y \leq G \ltimes^{\theta} Y$ has property (T).

\section{Rigid inclusions of von Neumann algebras}

Suppose that $G$ is a discrete pmp groupoid. One can then consider the Hilbert bundle $\mathcal{H}=$ $\bigsqcup_{x \in G^{0}} \ell^{2}(x G)$. Observe that one can canonically identify $L^{2}\left(G^{0}, \mathcal{H}\right)$ with $L^{2}(G)$. The left regular representation of $G$ is the representation $\lambda$ of $G$ on $\mathcal{H}$ defined as follows. For $\gamma \in G$ let $\delta_{\gamma} \in \ell^{2}(r(\gamma) G)$ be the indicator function of $\{\gamma\} \subset r(\gamma) G$. Then $\lambda_{\rho} \delta_{\gamma}=\delta_{\rho \gamma}$ for $(\rho, \gamma) \in G^{2}$. This gives rise the representation $[[\lambda]]$ of $[[G]]$ on $L(G)$. The groupoid von Neumann algebra of $G$ is defined to be the von Neumann algebra $L(G) \subset B\left(L^{2}(G)\right)$ generated by the elements $u_{\sigma}:=[[\lambda]]_{\sigma}$ for $\sigma \in[[G]]$. The main goal of this section is to provide a characterization of property $(\mathrm{T})$ for (triples of) groupoids in terms of the associated groupoid von Neumann algebra.

3.1. Hilbert bimodules and ucp maps. Suppose that $(M, \tau)$ is a tracial von Neumann algebra. We let $L^{2}(M)$ be the Hilbert space obtained from $(M, \tau)$ via the GNS construction, and $M \rightarrow L^{2}(M)$, $x \mapsto|x\rangle$ be the canonical inclusion. Thus $|1\rangle$ is the canonical cyclic vector of $M$.

A (Hilbert) $M$-bimodule is a Hilbert space $\mathfrak{H}$ endowed with commuting normal *-representations $\pi$ of $M$ and $\rho$ of $M^{\text {op }}$ on $\mathfrak{H}$. In this case, given $x, y \in M$ and $\xi \in \mathfrak{H}$, one writes $x \xi y$ for $\pi(x) \rho\left(y^{\text {op }}\right) \xi$. A vector $\xi$ of $\mathfrak{H}$ is called tracial if it satisfies $\langle\xi, a \xi\rangle=\tau(a)$ and $\langle\xi, \xi b\rangle=\tau(b)$ for every $a \in M$ and $b \in N$. Given a subset $F$ of $M$ and $\varepsilon>0$, a vector $\xi$ in $\mathfrak{H}$ is $F$-central if it satisfies $a \xi=\xi a$ for $a \in F$, and $(F, \varepsilon)$-central if it satisfies $\|a \xi-\xi a\| \leq \varepsilon$ for $a \in F$. The adjoint $M$-bimodule $\overline{\mathfrak{H}}$ is equal to the conjugate Hilbert space of $\mathfrak{H}$ endowed with the bimodule structure given by $x \bar{\xi} y=\overline{y^{*} \xi x^{*}}$ for $x, y \in M$ and $\xi \in \mathfrak{H}$.

A linear map $\phi: M \rightarrow M$ is completely positive (cp) if, for every $n \in \mathbb{N}, \operatorname{id}_{M_{n}(\mathbb{C})} \otimes \phi: M_{n}(\mathbb{C}) \otimes M \rightarrow$ $M_{n}(\mathbb{C}) \otimes N$ maps positive elements to positive elements. If furthermore $\phi(1)=1$, then $\phi$ is unital completely positive (ucp). A map $\phi: M \rightarrow M$ is trace-preserving if $\tau \circ \phi=\tau$. If $A$ is a subalgebra of $M$, then a map $\phi: M \rightarrow M$ is an $A$-bimodule map if it satisfies $\phi(a x)=a \phi(x)$ and $\phi(x a)=\phi(x) a$ for $x \in M$ and $a \in A$. Suppose that $\phi: M \rightarrow M$ is a cp $A$-bimodule map satisfying $\tau \circ \phi \leq \tau$ and $\phi(1) \leq 1$. Setting $T_{\phi}|x\rangle=|\phi(x)\rangle$ for $x \in M$ defines a bounded operator $T_{\phi}$ on $L^{2}(M)$. The adjoint $T_{\phi}^{*}$ of $T_{\phi}$ is of the form $T_{\phi^{*}}$, where $\phi^{*}: M \rightarrow M$ is a cp $A$-bimodule map satisfying $\tau \circ \phi^{*} \leq \tau$ and $\phi^{*}(1) \leq 1$; see [17, Lemma 1.2.1].

Given a nonzero normal cp $A$-bimodule map $\phi: M \rightarrow M$ satisfying $\phi(1) \leq 1$ and $\tau \circ \phi \leq \tau$, one can define a Hilbert $M$-bimodule associated with $\phi$ as follows. Consider the completion $\mathfrak{H}_{\phi}$ of $M \odot M$ with respect to the inner product defined by

$$
\langle a \otimes b, c \otimes d\rangle=\tau\left(b^{*} \phi\left(a^{*} c\right) d\right) .
$$

The $M$-bimodule structure is induced by the maps

$$
x(a \otimes b) y=x a \otimes b y
$$

for $x, a \in M$ and $b, y \in N$. Denoting by $\xi_{\phi}$ the vector of $\mathfrak{H}_{\phi}$ obtained from $1 \otimes 1$ one has that

$$
\left\langle b \xi_{\phi} x, a \xi_{\phi} y\right\rangle=\langle b \otimes x, a \otimes y\rangle=\tau\left(x^{*} \phi\left(b^{*} a\right) y\right)
$$


In particular we have that $\left\langle\xi_{\phi}, \xi_{\phi} \cdot\right\rangle=\tau(\phi(1) \cdot) \leq \tau$ and $\left\langle\xi_{\phi}, \cdot \xi_{\phi}\right\rangle=\tau \circ \phi \leq \tau$. Since $\phi$ is an $A$-bimodule map, $\xi_{\phi}$ is $A$-central. The vector $\xi_{\phi}$ is cyclic for $\mathfrak{H}_{\phi}$, in the sense that $\left\{a \xi_{\phi} b: a, b \in M\right\}$ has dense linear span in $\mathfrak{H}_{\phi}$. If $\phi$ is ucp and trace-preserving, then $\xi_{\phi}$ is a tracial unit vector.

Conversely, suppose that $\mathfrak{H}$ is an $M$-bimodule with an $A$-central cyclic vector $\xi$ satisfying $\langle\xi, \cdot \xi\rangle \leq \tau$ and $\langle\xi, \xi \cdot\rangle \leq \tau$. One can define a normal cp $A$-bimodule map $\phi: M \rightarrow M$ by setting $\phi(x)=L_{\xi}^{*} x L_{\xi}$. Here $L_{\xi}: L^{2}(M) \rightarrow \mathfrak{H}$ is the operator defined by $L_{\xi}|y\rangle=\xi y$ for $y \in M$. If $\xi$ is a tracial unit vector, then $\phi$ is a ucp trace-preserving map. These constructions are inverse of each other. More information on the correspondence between cp maps and Hilbert bimodules can be found in [17, Section 1].

3.2. Completely positive maps and functions of positive type. Let $G$ be a discrete pmp groupoid with unit space $X$. For a function of positive type $\varphi$ on a discrete pmp groupoid $G$ and $\sigma \in[[G]]$, denote by $\varphi(\sigma) \in L^{\infty}(X)$ the function

$$
x \mapsto\left\{\begin{array}{cc}
\varphi(x \sigma) & \text { if } x \in \operatorname{ran}(\sigma) \\
0 & \text { otherwise. }
\end{array}\right.
$$

The proof of the following proposition is similar to the proofs of $[9$, Lemma 1.1] and [1, Proposition 3.5.4]. Recall that, if $\sigma \in[[G]]$, then we let $u_{\sigma}$ be the element $[[\lambda]]_{\sigma}$ of $L(G) \subset B\left(L^{2}(G)\right)$, where $\lambda$ is the left regular representation of $G$.

Proposition 3.1. Suppose that $G$ is a discrete pmp group, $K \leq G$ is a subgroupoid, and $\varphi$ is a normalized $K$-invariant function of positive type on $G$. Then there exists a unique trace-preserving, $L(K)$-bimodule normal ucp map $\phi: L(G) \rightarrow L(G)$ such that

$$
\phi\left(u_{\sigma}\right)=\varphi(\sigma) u_{\sigma}
$$

for $\sigma \in[G]$.

Proof. Consider the GNS representation $\left(\pi^{\varphi}, \mathcal{H}^{\varphi}, \xi^{\varphi}\right)$ of $G$ associated with $\varphi$. Choose an orthonormal basic sequence $\left(e^{(i)}\right)_{i \in \mathbb{N}}$ for $\mathcal{H}^{\varphi}$. Define $a^{(i)} \in L^{\infty}(G)$ by setting

$$
a_{\gamma}^{(i)}:=\left\langle\xi_{r(\gamma)}^{\varphi}, \pi_{\gamma}^{\varphi} e_{s(\gamma)}^{(i)}\right\rangle=\left\langle\pi_{\gamma}^{\varphi *} \xi_{r(\gamma)}^{\varphi}, e_{s(\gamma)}^{(i)}\right\rangle .
$$

For $\gamma, \rho \in G$ one has that

$$
\begin{aligned}
\sum_{i \in \mathbb{N}} \bar{a}_{\rho}^{(i)} a_{\gamma}^{(i)} & =\sum_{i \in \mathbb{N}}\left\langle e_{s(\rho)}^{(i)}, \pi_{\rho}^{\varphi *} \xi_{r(\rho)}^{\varphi}\right\rangle\left\langle\pi_{\gamma}^{\varphi *} \xi_{r(\gamma)}^{\varphi}, e_{s(\gamma)}^{(i)}\right\rangle \\
& =\sum_{i, j \in \mathbb{N}}\left\langle e_{s(\rho)}^{(i)}, \pi_{\rho}^{\varphi *} \xi_{r(\rho)}^{\varphi}\right\rangle\left\langle\pi_{\gamma}^{\varphi *} \xi_{r(\gamma)}^{\varphi}, e_{s(\gamma)}^{(j)}\right\rangle\left\langle e_{s(\gamma)}^{(i)}, e_{s(\gamma)}^{(j)}\right\rangle \\
& =\sum_{i, j \in \mathbb{N}}\left\langle\left\langle\pi_{\rho}^{\varphi *} \xi_{r(\rho)}^{\varphi}, e_{s(\rho)}^{(i)}\right\rangle e_{s(\gamma)}^{(i)},\left\langle\pi_{\gamma}^{\varphi *} \xi_{r(\gamma)}^{\varphi}, e_{s(\gamma)}^{(j)}\right\rangle e_{s(\gamma)}^{(j)}\right\rangle \\
& =\left\langle\pi_{\rho}^{\varphi *} \xi_{r(\rho)}^{\varphi}, \pi_{\gamma}^{\varphi *} \xi_{r(\gamma)}^{\varphi}\right\rangle \\
& =\left\langle\xi_{r(\gamma)}^{\varphi}, \pi_{\rho \gamma^{-1}}^{\varphi} \xi_{r(\gamma)}^{\varphi}\right\rangle \\
& =\varphi\left(\rho \gamma^{-1}\right) .
\end{aligned}
$$

For $T \in L(G) \subset B\left(L^{2}(G)\right)$ set $\phi(T)=\sum_{i \in \mathbb{N}} a^{(i) *} T a^{(i)}$. The convergence is in strong operator topology, since $\sum_{i=1}^{n} a_{i}^{*} a_{i} \leq 1$ for every $n \in \mathbb{N}$.

Now for $\xi \in L^{2}(G)$ and $i \in \mathbb{N}$ we have that

$$
\begin{aligned}
\left(a^{(i) *} b u_{\sigma} a^{(i)} \xi\right)_{\gamma} & =\bar{a}_{\gamma}^{(i) *}\left(b u_{\sigma} a^{(i)} \xi\right)_{\gamma} \\
& =\bar{a}_{\gamma}^{(i) *} b_{r(\gamma)}\left(u_{\sigma} a^{(i)} \xi\right)_{\gamma} \\
& =\bar{a}_{\gamma}^{(i) *} b_{r(\gamma)}\left(a^{(i)} \xi\right)_{\sigma^{-1} \gamma} \\
& =\bar{a}_{\gamma}^{(i) *} b_{r(\gamma)} a_{\sigma^{-1} \gamma}^{(i)} \xi_{\sigma^{-1} \gamma}
\end{aligned}
$$


if $r(\gamma) \in \sigma \sigma^{-1}$, and $\left(a^{(i) *} b u_{\sigma} a^{(i)} \xi\right)_{\gamma}=0$ otherwise. Therefore we have that

$$
\begin{aligned}
\left(\phi\left(b u_{\sigma}\right) \xi\right)_{\gamma} & =\sum_{i \in \mathbb{N}}\left(\left(a^{(i)}\right)^{*} b u_{\sigma} a^{(i)} \xi\right)_{\gamma} \\
& =\sum_{i \in \mathbb{N}}\left(\left(\bar{a}_{\gamma}^{(i) *} a_{\sigma^{-1} \gamma}^{(i)}\right) b_{r(\gamma)} \xi_{\sigma^{-1} \gamma}\right. \\
& =\varphi(r(\gamma) \sigma) b_{r(\gamma)} \xi_{\sigma^{-1} \gamma} \\
& =\left(\varphi(\sigma) b u_{\sigma} \xi\right)_{\gamma}
\end{aligned}
$$

if $r(\gamma) \in \sigma \sigma^{-1}$, and

$$
\left(\phi\left(b u_{\sigma}\right) \xi\right)_{\gamma}=0=\left(\varphi(\sigma) b u_{\sigma} \xi\right)_{\gamma}
$$

otherwise. This shows that $\phi\left(b u_{\sigma}\right)=\varphi(\sigma) b u_{\sigma}$. Since $\sum_{i \in \mathbb{N}} a_{i}^{*} a_{i}=1$, we have that $\phi$ is unital. Clearly, $\phi$ is normal and completely positive, and hence completely contractive. If $\sigma^{\prime} \in[K]$ then we have that, since $\varphi$ is $K$-invariant,

$$
\phi\left(b u_{\sigma} u_{\sigma^{\prime}}\right)=\phi\left(b u_{\sigma \sigma^{\prime}}\right)=\varphi\left(\sigma \sigma^{\prime}\right) b u_{\sigma \sigma^{\prime}}=\varphi(\sigma) b u_{\sigma} u_{\sigma^{\prime}}=\phi\left(b u_{\sigma}\right) u_{\sigma^{\prime}}
$$

and

$$
\phi\left(u_{\sigma^{\prime}} b u_{\sigma}\right)=\phi\left(\theta_{\sigma^{\prime}}(b) u_{\sigma^{\prime} \sigma}\right)=\varphi\left(\sigma^{\prime} \sigma\right) \theta_{\sigma^{\prime}}(b) u_{\sigma^{\prime}} u_{\sigma}=u_{\sigma^{\prime}} \phi\left(b u_{\sigma}\right) .
$$

Similarly, if $a \in L^{\infty}(X) \subset L(G)$ one has that

$$
\phi\left(a b u_{\sigma}\right)=a \phi\left(b u_{\sigma}\right)
$$

and

$$
\phi\left(b u_{\sigma} a\right)=\phi\left(b u_{\sigma}\right) a .
$$

These equations together with that fact that $\phi$ is a normal ucp map imply that $\phi$ is an $L(K)$-bimodule map. This concludes the proof.

3.3. Rigidity for von Neumann algebras. In order to characterize property $(\mathrm{T})$ for triples of groupoids, we introduce a notion of rigidity for a nested quadruple of von Neumann algebra.

Definition 3.2. Let $(M, \tau)$ be a tracial von Neumann algebra and $Z \subset A \subset B \subset M$ be von Neumann subalgebras. Then the quadruple $Z \subset A \subset B \subset M$ is rigid if for every $\varepsilon>0$ and nonzero projection $p_{0} \in Z$ there is a finite subset $F$ of $M$ and $\delta>0$ such that for any Hilbert $M$-bimodule $\mathfrak{H}$ with an $(F, \delta)$-central and $A$-central tracial unit vector $\xi \in \mathfrak{H}$ there is a nonzero projection $p \in Z$ such that $p \leq p_{0}$ and for every projection $q \in Z$ such that $q \leq p$ one has that $\|x \xi-\xi x\| \leq \tau(q)^{1 / 2}\|x\| \varepsilon$ for $x \in q B q$.

As in the case of rigidity for pairs of von Neumann algebras as defined in [17], one can provide several equivalent characterizations of rigidity for quadruples $Z \subset A \subset B \subset M$. The proof of this fact is standard, and follows arguments from $[10,14,15,17]$. We present a full proof, for the reader's convenience.

Proposition 3.3. Let $(M, \tau)$ be a tracial von Neumann algebra and $Z \subset A \subset B \subset M$ be von Neumann subalgebras. The following assertions are equivalent:

(1) $Z \subset A \subset B \subset M$ is rigid;

(2) for every $\varepsilon>0$ and nonzero projection $p_{0} \in Z$ there exist a finite subset $F$ of $M$ and $\delta>0$ such that if $\phi: M \rightarrow M$ is a normal trace-preserving ucp A-bimodule map such that $\max _{x \in F}\|\phi(x)-x\|_{2} \leq \delta$, then there exists a nonzero projection $p \in Z$ such that $p \leq p_{0}$ and for every projection $q \in Z$ such that $q \leq p$ one has that $\|\phi(b)-b\|_{2} \leq \varepsilon \tau(q)^{1 / 2}\|b\|$ for every $b \in q B q$.

(3) for every $\varepsilon>0$ and nonzero projection $p_{0} \in Z$ there is a finite subset $F$ of $M$ and $\delta>0$ such that if $\phi: M \rightarrow M$ is a normal cp A-bimodule map such that $\tau \circ \phi \leq \tau, \phi(1) \leq 1, \phi=\phi^{*}$, and $\max _{x \in F}\|\phi(x)-x\|_{2} \leq \delta$, then there exists a nonzero projection $p \in Z$ such that $p \leq p_{0}$ and for every projection $q \in Z$ such that $q \leq p$ one has that $\|\phi(b)-b\|_{2} \leq \varepsilon \tau(q)^{1 / 2}\|b\|$ for every $b \in q B q$; 
(4) for every $\varepsilon>0$ and nonzero projection $p_{0} \in Z$ there is a finite subset $F$ of $M$ and $\delta>0$ such that if $\phi: M \rightarrow M$ is a normal cp A-bimodule map such that $\tau \circ \phi \leq \tau, \phi(1) \leq 1$, and $\max _{x \in F}\|\phi(x)-x\|_{2} \leq \delta$, then there exists a nonzero projection $p \in Z$ such that $p \leq p_{0}$ and for every projection $q \in Z$ such that $q \leq p$ one has that $\|\phi(b)-b\|_{2} \leq \varepsilon \tau(q)^{1 / 2}\|b\|$ for every $b \in q B q$

(5) for every $\varepsilon>0$ and nonzero projection $p_{0} \in Z$ there is a finite subset $F$ of $M$ and $\delta>0$ such that if $\mathfrak{H}$ is an $M$-bimodule and $\xi \in \mathfrak{H}$ is an $(F, \delta)$-central and $A$-central unit vector satisfying $\langle\xi, \cdot \xi\rangle \leq \tau$ and $\langle\xi, \xi \cdot\rangle \leq \tau$, then there exists a nonzero projection $p \in Z$ such that $p \leq p_{0}$ and for every projection $q \in Z$ such that $q \leq p$ one has that $\|x \xi-\xi x\| \leq \tau(q)^{1 / 2}\|x\| \varepsilon$ for every $x \in q B q$.

Proof. (1) $\Rightarrow(2)$ Let $F$ be a finite subset of $M$, and $\delta>0$. Suppose that $\phi: M \rightarrow M$ is a normal trace-preserving ucp $A$-bimodule map such that $\max _{x \in F}\|x\|_{2}\|\phi(x)-x\|_{2} \leq \delta / 2$. Let $(\mathfrak{H}, \xi)$ be the corresponding Hilbert $M$-bimodule with distinguished $A$-central tracial unit vector $\xi$. For $x \in M$ we have that

$$
\begin{aligned}
\|x \xi-\xi x\|^{2} & =\|x \xi\|^{2}+\|\xi x\|^{2}-2 \operatorname{Re}\langle x \xi, \xi x\rangle \\
& =2\|x\|_{2}^{2}-2 \operatorname{Re}\langle\phi(x), x\rangle_{L^{2}(M)} \\
& =2 \operatorname{Re}\langle x-\phi(x), x\rangle_{L^{2}(M)} \\
& \leq 2\|x-\phi(x)\|_{2}\|x\|_{2} \leq \delta .
\end{aligned}
$$

By assumption, one can choose $F$ and $\delta \leq \varepsilon$ in such a way that this guarantees the existence of nonzero projection $p \in Z$ such that $p \leq p_{0}$ and $\|x \xi-\xi x\|_{2} \leq \tau(q)^{1 / 2}\|x\| \varepsilon$ for every projection $q \in Z$ such that $q \leq p$, and for $x \in q B q$. For such a $q \in Z$ and $x \in q B q$ we have that

$$
\begin{aligned}
\|\phi(x)-x\|_{2}^{2} & =\|\phi(x)\|_{2}^{2}+\|x\|_{2}^{2}-2 \operatorname{Re} \tau\left(\phi(x)^{*} x\right) \\
& \leq(\tau \circ \phi)\left(x^{*} x\right)+\tau\left(x^{*} x\right)-2 \operatorname{Re} \tau\left(\phi(x)^{*} x\right) \\
& =\|x \xi-\xi x\|^{2} \leq \tau(q)\|x\|^{2} \varepsilon^{2} .
\end{aligned}
$$

$(2) \Rightarrow(3)$ Suppose that $\phi: M \rightarrow M$ is a ucp map such that $\tau \circ \phi \leq \tau, \phi(1) \leq 1, \phi=\phi^{*}$, and $\|\phi(1)-1\|_{2} \leq \delta$. Since $T_{\phi}^{*}=T_{\phi^{*}}=T_{\phi}$, we have that $\tau(x \phi(y))=\tau(\phi(x) y)$ for $x, y \in M$. Consider then the ucp map $\psi: M \stackrel{\phi}{\rightarrow} M$ defined by

$$
\psi(x)=\phi(x)+(1-(\tau \circ \phi)(1)) \mathrm{E}_{Z}(x)
$$

where $\mathrm{E}_{Z}: M \rightarrow Z \subset M$ is the canonical trace-preserving conditional expectation. Observe that $T_{\mathrm{E}_{Z}}=\mathrm{e}_{Z}: L^{2}(M) \rightarrow L^{2}(Z) \subset L^{2}(M)$ is the orthogonal projection. Therefore we have that $\tau\left(x \mathrm{E}_{Z}(y)\right)=\tau\left(\mathrm{E}_{Z}(x) y\right)$ for every $x, y \in M$. Thus $\tau(x \psi(y))=\tau(\psi(x) y)$. Furthermore we have that $(\tau \circ \psi)(1)=1$. From this we deduce that

$$
\begin{aligned}
\|\psi(1)-1\|_{2}^{2} & =\|\psi(1)\|_{2}^{2}+1-2 \operatorname{Re} \tau(\psi(1)) \\
& =\tau\left(\psi(1)^{2}\right)-1 \leq \tau(\psi(1))-1=0 .
\end{aligned}
$$

Thus $\psi$ is unital, which implies that $\psi$ is trace-preserving.

Observe now that since $\|\phi(1)-1\|_{2} \leq \delta$, we have $|1-(\tau \circ \phi)(1)| \leq \delta$. Therefore for a projection $q \in Z$ and $b \in q M q$ we have that

$$
\|\psi(b)-\phi(b)\|_{2} \leq \tau(q)^{1 / 2} \delta\|b\| .
$$

This easily gives the desired implication.

$(3) \Rightarrow(4)$ Suppose that $\phi: M \rightarrow M$ is a normal ucp $A$-bimodule map such that $\tau \circ \phi \leq \tau, \phi(1) \leq 1$. Observe then that $\psi:=\frac{1}{2}\left(\phi+\phi^{*}\right): M \rightarrow M$ is a normal cp $A$-bimodule map satisfying $\psi(1) \leq 1$, $\tau \circ \psi \leq \tau, \psi=\psi^{*}$. Furthermore for a projection $q \in Z$ and a unitary $u \in q M q$ one has that

$$
\|\psi(u)-u\|_{2} \leq \frac{1}{2}\|\phi(u)-u\|_{2}+\frac{1}{2}\left\|\phi^{*}(u)-u\right\|_{2} \leq 2\|\phi(u)-u\|_{2}^{\frac{1}{2}}
$$


by [17, Lemma 1.1.5]. Since every element $x$ of $q M q$ with $\|x\|<1$ is a convex combination of unitaries, this suffices; see also [15, Lemma 3].

$(4) \Rightarrow(5)$ Let $F$ be a finite subset of the unitary group of $M$ containing 1 , and $\delta>0$. Suppose that $\mathfrak{H}$ is an $M$-bimodule and $\xi \in M$ is an $A$-central and $(F, \delta)$-central unit vector satisfying $\langle\xi, \cdot \xi\rangle \leq \tau$ and $\langle\xi, \xi \cdot\rangle \leq \tau$. We can assume that $\xi$ is cyclic. Consider the normal cp map $\phi: M \rightarrow M$ associated with $(\mathfrak{H}, \xi)$. This is defined by $\phi(x)=L_{\xi}^{*} x L_{\xi}$, where $L_{\xi}|x\rangle=\xi x$. Then we have that $\phi$ is a normal $A$-module cp map satisfying

$$
(\tau \circ \phi)(x)=\left\langle 1\left|L_{\xi}^{*} x L_{\xi}\right| 1\right\rangle=\langle\xi \mid x \xi\rangle \leq \tau(x) .
$$

Furthermore

$$
\langle x|\phi(1)| x\rangle=\left\langle x\left|L_{\xi}^{*} L_{\xi}\right| x\right\rangle=\langle\xi x \mid \xi x\rangle=\left\langle\xi \mid \xi x x^{*}\right\rangle \leq \tau\left(x x^{*}\right)=\|x\|_{2}^{2}=\langle x \mid x\rangle
$$

and thus $\phi(1) \leq 1$. We have that $(\tau \circ \phi)(1)=\langle\xi \mid \xi\rangle=1$ since $\xi$ is a unit vector. For $u \in F$,

$$
\begin{aligned}
\|\phi(u)-u\|_{2}^{2} & =\|\phi(u)\|_{2}^{2}+1-2 \operatorname{Re} \tau\left(\phi(u)^{*} u\right) \\
& \leq 2-2 \operatorname{Re} \tau\left(\phi(u)^{*} u\right) \\
& =\|u \xi-\xi u\|^{2} \leq \delta
\end{aligned}
$$

By assumption, choosing $F$ large enough and $\delta$ small enough guarantees that there exists a nonzero projection $p \in Z$ such that $p \leq p_{0}$ and, for every projection $q \in Z$ such that $q \leq p$ and $x \in q B q$, $\|\phi(x)-x\|_{2} \leq \tau(q)^{1 / 2}\|x\| \varepsilon$. This implies that, for a unitary $u \in U(q B q)$,

$$
\begin{aligned}
\|u \xi-\xi u\|^{2} & =(\tau \circ \phi)(q)+\tau(q)-2 \operatorname{Re} \tau\left(\phi(u)^{*} u\right) \\
& \leq 2 \tau(q)-2 \operatorname{Re} \tau\left(\phi(u)^{*} u\right) \\
& =2 \operatorname{Re} \tau\left(q\left(q-q u \phi(u)^{*}\right)\right) \\
& \leq 2 \tau(q)^{1 / 2}\left\|q-q u \phi(u)^{*}\right\|_{2} \\
& \leq 2 \tau(q) \varepsilon .
\end{aligned}
$$

Therefore $\|u \xi-\xi u\| \leq \tau(p)^{1 / 2} 2 \varepsilon^{1 / 2}$ for a unitary $u$ in $q B q$. Since every element $b \in q B q$ with $\|b\|<1$ is a convex combination of unitaries, this concludes the proof.

$(5) \Rightarrow(1)$ Obvious.

Remark 3.4. Proposition 3.3 shows that, if $M$ is a von Neumann algebra and $B \subset M$ is a subalgebra, then $B \subset M$ is rigid in the sense of [17, Definition 4.2.1] if and only if $\mathbb{C} 1 \subset \mathbb{C} 1 \subset B \subset M$ is rigid in the sense of Definition 3.2; see also [15, Theorem 1] and [10, Theorem 3.1].

3.4. von Neumann algebra characterization of property $(\mathbf{T})$ for groupoids. Now we use the characterization of property $(\mathrm{T})$ for groupoids from Theorem 2.13 together with the characterization of rigidity for inclusions of von Neumann algebras from Proposition 3.3 to give a characterization of property $(\mathrm{T})$ for groupoids in terms of the corresponding groupoid von Neumann algebra.

Theorem 3.5. Suppose that $G$ is a discrete pmp groupoid, and $K \leq H \leq G$ are subgroupoid. Assume that $H$ is ergodic. Let $X$ be the common unit space of $K, H, G$. The following assertions are equivalent:

(1) $K \leq H \leq G$ has property (T);

(2) for every $\varepsilon>0$ and nonzero projection $p_{0} \in L^{\infty}(X)$ there exist a finite subset $F$ of $L(G)$ and $\delta>0$ such that for every Hilbert $M$-bimodule $\mathfrak{H}$ with an $(F, \delta)$-central and $L(K)$-central tracial unit vector $\xi \in \mathfrak{H}$ there is a nonzero projection $p \in Z$ and an $H$-central vector $\eta \in \mathfrak{H}$ such that $p \leq p_{0}$ and $\|q \eta-q \xi\| \leq \tau(q)^{1 / 2} \varepsilon$ for every projection $q \in L^{\infty}(X)$ such that $q \leq p ;$

(3) the inclusion $L^{\infty}(X) \subset L(K) \subset L(H) \subset L(G)$ is rigid;

(4) for every $\varepsilon>0$ there is a finite subset $F$ of $L(G)$ and $\delta>0$ such that for any Hilbert $M$ bimodule $\mathfrak{H}$ with an $(F, \delta)$-central and $L(K)$-central tracial unit vector $\xi \in \mathfrak{H}$ there is a nonzero projection $p \in L^{\infty}(X)$ such that $\|x \xi-\xi x\| \leq \tau(p)^{1 / 2}\|x\| \varepsilon$ for $x \in p L(H) p$. 
Proof. (1) $\Rightarrow(2)$ Suppose that $K \leq H \leq G$ has property (T). Fix $\varepsilon>0$ and a nonzero projection $p_{0} \in L^{\infty}(X)$. Then $p_{0}$ can be seen as the characteristic function of some Borel subset $B$ of $X$. Let $F$ be a finite subset of $[G]$ and $\delta>0$ be obtained from $\varepsilon$ and $B$ via Item (7) of the characterization of property $(\mathrm{T})$ for triples of groupoids provided by Theorem 2.13. Let now $\mathfrak{H}$ be an $L(G)$-bimodule with an $L(K)$-central and $(F, \delta)$-central tracial unit vector $\xi^{0} \in L$. We can assume that $\xi^{0}$ is a cyclic vector for $\mathfrak{H}$. The assignment $a \mapsto(\xi \mapsto a \xi)$ defines a normal *-representation of $L^{\infty}(X)$ on $\mathfrak{H}$. Thus there is a Hilbert bundle $\mathcal{H}=\left(\mathcal{H}_{x}\right)_{x \in X}$ such that $\mathfrak{H}=L^{2}(X, \mathcal{H})$ and, for $\xi=\left(\xi_{x}\right)_{x \in X} \in L^{2}(X, \mathcal{H})$ and $a=\left(a_{x}\right)_{x \in X} \in L^{\infty}(X), a \xi=\left(a_{x} \xi_{x}\right)_{x \in X}$; see [12, Theorem 14.2.1] or [21, Proposition F.26]. Suppose now that $t \in[G]$ and $a \in L^{\infty}(X)$. Observe that $u_{t} a=\theta_{t}(a) u_{t}$ where $\theta_{t}(a)=\left(a_{s(x t)}\right)_{x \in X} \in$ $L^{\infty}(X)$. Therefore we have that $u_{t} a \xi u_{t}^{*}=\theta_{t}(a) u_{t} \xi u_{t}^{*}$. This shows that the operator $\xi \mapsto u_{t} \xi u_{t}^{*}$ on $L^{2}(X, \mathcal{H})$ intertwines the normal *-representations $a \mapsto(\xi \mapsto a \xi)$ and $a \mapsto\left(\xi \mapsto \theta_{t}(a) \xi\right)$ of $L^{\infty}(X)$ on $\mathfrak{H}$. Therefore $\xi \mapsto u_{t} \xi u_{t}^{*}$ is a decomposable operator; see [20, Theorem 7.10], [21, Theorem F.21], or $\left[8\right.$, Subsection 2.5]. This means that exists a section $x \mapsto \pi_{x t} \in B\left(H_{s(x t)}, H_{x}\right)$ such that $u_{t} \xi u_{t}^{*}=$ $\left(\pi_{x t} \xi_{s(x t)}\right)_{x \in X}$ for $\xi \in L^{2}\left(X,\left(\mathcal{H}_{x}\right)_{x \in X}\right)$; see [20, Definition 7.9]. By considering such a decomposition when $t$ varies within a countable dense subgroup of $[G]$, and by the essential uniqueness of such a decomposition [21, Proposition F.33], one obtains a representation $\gamma \mapsto \pi_{\gamma}$ of $G$ on $\mathcal{H}$ such that $u_{t} \xi u_{t}^{*}=[\pi]_{t} \xi$ for every $t \in[G]$ and $\xi \in L^{2}(X, \mathcal{H})$. Since by assumption $\xi^{0}$ is $L(K)$-central, we have that $\xi^{0}$ is $K$-invariant. Furthermore since $\xi^{0}$ is $(F, \delta)$-central, we have that $\xi^{0}$ is $(F, \delta)$-invariant for $\pi$. Therefore by the choice of $F$ and $\delta$, there exists a non-null Borel subset $A$ of $X$ and an element $\eta$ of $L^{2}(X, \mathcal{H})$ such that $\eta$ is $H$-invariant and $\left\|\eta_{x}-\xi_{x}^{0}\right\| \leq \varepsilon$ for every $x \in A$. The vector $\eta$ together with the characteristic function $p$ of $A$ witness that the desired conclusion holds.

$(2) \Rightarrow(3)$ Suppose that $M$ is a tracial von Neumann algebra, $Z \subset M$ is a subalgebra, $q \in Z$ is a projection, and $\mathfrak{H}$ is an $M$-bimodule. Consider a $Z$-central unit vector $\xi \in \mathfrak{H}$ and an $M$-central vector $\eta \in \mathfrak{H}$ such that $\|q \xi-q \eta\| \leq \tau(q)^{1 / 2} \varepsilon$. Then for every unitary $u$ in $q M q$ one has that

$$
\|u \xi-\xi u\| \leq\|u \xi-u \eta\|+\|\xi u-\eta u\| \leq 2\|q \xi-q \eta\| .
$$

One can easily prove the implication $(2) \Rightarrow(3)$ using this observation.

$(3) \Rightarrow(4)$ This is obvious.

$(4) \Rightarrow(1)$ Suppose that (4) holds. We verify that Item (8) of Theorem 2.13 holds. To this purpose, fix $\varepsilon>0$. We want to find a finite subset $Q$ of $[G]$ and $\delta>0$ such that, for every normalized $K$-invariant function of positive type $\varphi$ on $G$ such that $\max _{t \in Q} \int_{x \in X}|\varphi(x t)-1|^{2} d \mu(x) \leq \delta$, there is a non-null Borel subset $A$ of $X$ such that $\operatorname{Re}(1-\varphi(\gamma)) \leq \varepsilon$ for a.e. $\gamma \in A H A$. Consider a finite subset $F$ of $L(G)$ and $\delta>0$ with the property that, for every trace-preserving ucp $L(K)$ bimodule map $\phi: L(G) \rightarrow L(G)$ satisfying $\max _{x \in F}\|\phi(x)-x\|_{2} \leq \delta$, there exists a nonzero projection $p \in L^{\infty}(X)$ such that $\|\phi(x)-x\|_{2} \leq \tau(p)^{1 / 2} \varepsilon\|x\|$ for $x \in p L(H) p$. By Kaplanski's density theorem [4, I.9.1.3], we can assume without loss of generality that there exists a finite subset $Q$ of $[G]$ such that $F=\left\{u_{t}: t \in Q\right\} \subset L(G)$. Consider a $K$-invariant normalized function of positive type $\varphi$ on $G$. Suppose that $\max _{t \in Q} \int_{x \in X}|\varphi(x t)-1| d \mu(x) \leq \delta$. Let $\phi: L(G) \rightarrow L(G)$ be the trace-preserving ucp $L(K)$-bimodule map associated with $\varphi$ as in Proposition 3.1. Observe that, for every $t \in Q$, $\phi\left(u_{t}\right)=\varphi(t) u_{t}$, where $\varphi(t) \in L^{\infty}(X)$ is the function $x \mapsto \varphi(x t)$. Therefore for $t \in Q$ we have that

$$
\left\|\phi\left(u_{t}\right)-u_{t}\right\|_{2}=\left\|\varphi(t) u_{t}-u_{t}\right\|_{2} \leq\|\varphi(t)-1\|_{2} \leq \delta .
$$

Therefore by assumption there exists a nonzero projection $p \in L^{\infty}(X)$ such that for every projection $q \in L^{\infty}(X)$ such that $q \leq p$ one has that $\|\phi(x)-x\|_{2} \leq \tau(q)^{1 / 2} \varepsilon\|x\|$ for $x \in q L(H) q$. Let now $A \subset X$ be a Borel subset such that $p$ is the characteristic function of $A$. Then we have that, for $\sigma \in[A H A]$, $u_{\sigma} \in p L(H) p, \tau(p)=\mu(A)$, and hence

$$
\mu(A) \varepsilon^{2} \geq\left\|\phi\left(u_{\sigma}\right)-u_{\sigma}\right\|_{2}^{2}=\|\varphi(\sigma)-1\|_{2}^{2}=\int_{x \in A}|\varphi(x \sigma)-1|^{2} d \mu .
$$

Since this holds for every $\sigma \in[A H A]$, we conclude that $|\varphi(\gamma)-1| \leq \varepsilon$ for a.e. $\gamma \in A H A$. This shows that Item (8) of the characterization of property $(\mathrm{T})$ for triples of groupoids holds. This concludes the proof. 


\section{REFERENCES}

1. Andreas Aaserud, Applications of property (T) for groups and von Neumann algebras, Ph.D. thesis, University of Copenhagen, 2011.

2. Claire Anantharaman-Delaroche, Cohomology of property $(T)$ groupoids and applications, Ergodic Theory and Dynamical Systems 25 (2005), no. 4, 977-1013.

3. Bachir Bekka, Pierre de la Harpe, and Alain Valette, Kazhdan's property (T), New Mathematical Monographs, vol. 11, Cambridge University Press, Cambridge, 2008.

4. Bruce Blackadar, Operator algebras, Encyclopaedia of Mathematical Sciences, vol. 122, SpringerVerlag, Berlin, 2006.

5. Alain Connes and Vaughan Jones, Property $T$ for von Neumann algebras, 17, no. 1, 57-62.

6. Pierre de la Harpe and Alain Valette, La propriété $(T)$ de Kazhdan pour les groupes localement compacts (avec un appendice de Marc Burger), Astérisque (1989), no. 175, 158.

7. Eusebio Gardella and Martino Lupini, The complexity of conjugacy, orbit equivalence, and von Neumann equivalence of actions of nonamenable groups, preprint.

8. — Representations of étale groupoids on $L_{p}$-spaces, arXiv:1408.3752 (2014).

9. Uffe Haagerup, An example of a nonnuclear $C^{*}$-algebra, which has the metric approximation property, Inventiones Mathematicae 50 (1978), no. 3, 279-293.

10. Adrian Ioana, Jesse Peterson, and Sorin Popa, Amalgamated free products of weakly rigid factors and calculation of their symmetry groups, Acta Mathematica 200, no. 1, 85-153.

11. Paul Jolissaint, On property (T) for pairs of topological groups, L'Enseignement Mathématique 51 (2005), no. 1-2, 31-45.

12. Richard V. Kadison and John R. Ringrose, Fundamentals of the theory of operator algebras. Vol. II, Pure and Applied Mathematics, vol. 100, Academic Press, Inc., Orlando, FL, 1986.

13. Alexander Kechris, Classical descriptive set theory, Graduate Texts in Mathematics, vol. 156, Springer-Verlag, New York, 1995.

14. Jesse Peterson, A 1-cohomology characterization of property $(T)$ in von Neumann algebras, 243 , no. $1,181-199$.

15. Jesse Peterson and Sorin Popa, On the notion of relative property (T) for inclusions of von Neumann algebras, Journal of Functional Analysis 219 (2005), no. 2, 469-483.

16. Sorin Popa, Correspondences, INCREST preprint (1986), unpublished.

17. Second Series 163 (2006), no. 3, 809-899.

18. Arlan Ramsay, Virtual groups and group actions, Advances in Mathematics 6 (1971), no. 3, 253322.

19. Jean Renault, $C^{*}$-algebras and dynamical systems, Publicações Matemáticas do IMPA, Instituto Nacional de Matemética Pura e Aplicada, Rio de Janeiro, 2009.

20. Masamichi Takesaki, Theory of operator algebras. I, Encyclopaedia of Mathematical Sciences, vol. 124, Springer-Verlag, Berlin, 2002.

21. Dana P. Williams, Crossed products of $C^{*}$-algebras, Mathematical Surveys and Monographs, vol. 134, American Mathematical Society, Providence, RI, 2007.

22. Robert J. Zimmer, On the cohomology of ergodic actions of semisimple Lie groups and discrete subgroups, American Journal of Mathematics 103 (1981), no. 5, 937-951.

Martino Lupini, Mathematics Department, California Institute of Technology, 1200 E. California Blvd, MC 253-37, PASAdena, CA 91125

E-mail address: lupini@caltech.edu

URL: http://www.lupini.org/ 